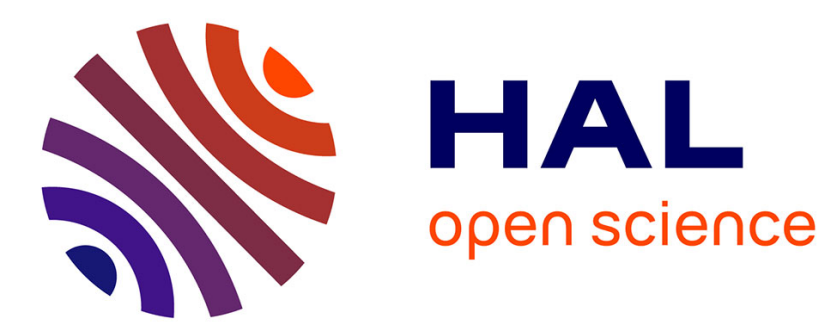

\title{
Rayleigh-Bénard convection in non-Newtonian fluids: Experimental and theoretical investigations
}

\author{
Mondher Bouteraa, Thomas Varé, Chérif Nouar, Simon Becker, Jamal
}

Ouhajjou

\section{- To cite this version:}

Mondher Bouteraa, Thomas Varé, Chérif Nouar, Simon Becker, Jamal Ouhajjou. Rayleigh-Bénard convection in non-Newtonian fluids: Experimental and theoretical investigations. Physics of Fluids, 2021, 33, pp.114112. 10.1063/5.0070983 . hal-03454578

\section{HAL Id: hal-03454578 \\ https://hal.science/hal-03454578}

Submitted on 29 Nov 2021

HAL is a multi-disciplinary open access archive for the deposit and dissemination of scientific research documents, whether they are published or not. The documents may come from teaching and research institutions in France or abroad, or from public or private research centers.
L'archive ouverte pluridisciplinaire HAL, est destinée au dépôt et à la diffusion de documents scientifiques de niveau recherche, publiés ou non, émanant des établissements d'enseignement et de recherche français ou étrangers, des laboratoires publics ou privés. 


\title{
Rayleigh-Bénard convection in non-Newtonian fluids: Experimental and theoretical investigations
}

\author{
Mondher Bouteraa, Thomas Varé, Chérif Nouar,* Simon Becker, and Jamal Ouhajjou \\ LEMTA UMR 7563 CNRS - Université de Lorraine \\ ENSEM, 2 Avenue de la Forêt de Haye, \\ TSA 60604 - 54516 Vandoeuvre lès Nancy cedex, France
}

\begin{abstract}
We present an experimental and theoretical study of Rayleigh-Bénard convection in shearthinning fluids with temperature dependent properties. Experiments were performed using a cylindrical cell of radius $\hat{R}=60 \mathrm{~mm}$ and height adjustable at $\hat{d}=15 \mathrm{~mm}$ and $20 \mathrm{~mm}$ giving a radius-toheight ratio $L=4$ and 3 respectively. The fluids used are glycerol (Newtonian fluid) and aqueous xanthan gum solutions (shear-thinning fluids) at $1000 \mathrm{ppm}$ and $1200 \mathrm{ppm}$. Convection patterns are visualized by the shadowgraph method. In the theoretical part of this study, the weakly nonlinear analysis performed by Thomas Varé et al. [J. Fluid Mech. 905, A33(2020)] is extended to take into account the variation of the thermal expansion coefficient with temperature.

For the xanthan gum solutions used, the temperature dependence of the fluid parameters is sufficiently strong to obtain hexagonal cells at the onset of convection. It has been observed that their size decreases with increasing the temperature difference across the fluid layer, above the critical value. This result provides an experimental support to our theoretical study where it is shown that for hexagons, the band of stable wavenumbers is bent towards higher wavenumbers. For the glycerol, Newtonian fluid with a large Prandtl number, a slight increase of the wavelength of rolls is observed in agreement with the literature.
\end{abstract}

\footnotetext{
* cherif.nouar@univ-lorraine.fr
} 


\section{INTRODUCTION}

Rayleigh-Bénard convection has served as an excellent paradigm to study competition between different patterns with different symmetries in non equilibrium nonlinear systems. From theoretical point of view, the interest has been mainly in the competition between the roll, square and hexagonal planforms (see for instance [1]). The main control parameter is the Rayleigh number $R a$ which represents the dimensionless temperature across the fluid layer and is a measure of the external stress applied to the system. Under Boussinesq approximations, the physical properties of the fluid are considered independent of the temperature except the density in the buoyancy term. In addition, the density is assumed to vary linearly as a function of temperature, i.e. the thermal coefficient expansion is assumed constant. With these assumptions, the pattern which forms at onset, $R a=R a_{c}$ consists of rolls and the bifurcation from a quiescent state to rolls is stationary and continuous, i.e. supercritical [2]. The first theoretical investigation of supercritical convection was made by Busse [3] for infinite Prandtl number and by Busse \& Clever [4] for moderate Prandtl number. It is shown that when $R a$ does not exceed a certain value (22600 for an infinite Prandtl number) there exists a finite band of $k$ values at which two dimensional rolls are stable. Very near the critical Rayleigh number, the band of stable wavenumbers shrinks and $k$ tends to $k_{c}$. When $k$ is outside this band, secondary disturbances grow such that the flow readjusts its wavenumber to enter into the stable band. Although a large number of periodicities are mathematically allowed, experiments started from uncontrolled initial conditions have shown that a much more narrow range of wavenumbers are observed in practice.

In the experiments of Krishnamurti [5], it was observed that the size of rolls increases with increasing $R a$ and this increase is more sensitive for fluids with low $\operatorname{Pr}$. Further evidence of an increasing wavelength (decreasing wavenumber) has been provided by Koschmieder [6], Koshmieder \& Pallas [7] and Willis et al. [8]. Thus, the domain $k>k_{c}$ of Busse's stability diagram is not selected by the nonlinear effects.

When the variation of the fluid properties (density, viscosity, thermal conductivity, thermal expansion, specific heat) vary sufficiently with the temperature, i.e. when non-OberbeckBoussinesq (NOB) effects become substantial, the up-down reflection symmetry with respect 
to the midplane of the fluid layer is strongly broken, leading to the formation of hexagons at $R a=R a_{a}$ via a transcritical bifurcation. Hexagons are preferred to rolls in the vicinity of onset. When $R a$ increases by a sufficient amount, say $R a>R a_{r}$, the weakly non linear analysis based on amplitude equations [9] predicts that convection rolls become a stable solution. Increasing further the Rayleigh number, hexagons lose their stability after some threshold $R a_{h}$ and only rolls remain stable. Thus in the range of Rayleigh numbers, $R a_{r} \leq R a \leq R a_{h}$, both rolls and hexagons are expected to be stable. The threshold values $R a_{a}, R a_{r}$ and $R a_{h}$ have been calculated by Busse [9] for a system of infinite lateral extent. Using the conventional distance from the onset

$$
\epsilon=\left(R a-R a_{c}\right) / R a_{c}
$$

the theoretical predictions of Busse [9] indicate that $\epsilon_{a}, \epsilon_{r}$ and $\epsilon_{h}$ are proportional to $Q^{2}$, where $Q=\sum_{i=0}^{i=4} \gamma_{i} \mathcal{P}_{i}$ is a nondimensional parameter introduced by Busse [9] to characterize the departure from Boussinesq approximations. The non-Boussinesq coefficients $\gamma_{i}$ are the nondimensional variations of density $\hat{\rho}$, thermal expansion $\hat{\chi}$, kinematic viscosity $\hat{\nu}$, thermal conductivity $\hat{k}$ and specific heat $\hat{C p}$ with respect to the top and bottom temperatures normalized by some reference value:

$$
\begin{aligned}
& \gamma_{0}=\frac{\hat{\rho}_{2}-\hat{\rho}_{1}}{\hat{\rho}_{0}}, \quad \gamma_{1}=\frac{\hat{\chi}_{1}-\hat{\chi}_{2}}{\hat{\chi}_{0}}, \quad \gamma_{2}=\frac{\hat{\nu}_{1}-\hat{\nu}_{2}}{\hat{\nu}_{0}}, \\
& \gamma_{3}=\frac{\hat{k}_{1}-\hat{k}_{2}}{2 \hat{k}_{0}}, \quad \gamma_{4}=\frac{\hat{C}_{p_{1}}-\hat{C}_{p_{2}}}{\hat{C}_{p_{0}}} .
\end{aligned}
$$

The subscripts 1,2 and 0 indicate fluid properties evaluated at bottom, top and mean temperature of the cell respectively. The coefficients $\mathcal{P}_{i}$ are linear functions of $\operatorname{Pr}^{-1}$ where $\operatorname{Pr}$ is the Prandtl number. They were first given by Busse [9] and recalculated after by Bodenshatz [1]. The following expressions are reported:

$$
\begin{aligned}
& \mathcal{P}_{0}=2.676-\frac{0.361}{\operatorname{Pr}}, \quad \mathcal{P}_{1}=-6.631-\frac{0.772}{\operatorname{Pr}}, \quad \mathcal{P}_{2}=2.765 \\
& \mathcal{P}_{3}=9.54, \quad \mathcal{P}_{4}=-6.225+\frac{0.386}{\operatorname{Pr}}
\end{aligned}
$$

Note that in the study of Busse [9], the coefficients $\gamma_{i}$ and $Q$ are evaluated at the onset of convection $(\epsilon=0)$. Correlations for the thresholds $\epsilon_{a}, \epsilon_{r}$ and $\epsilon_{h}$ as a function of $Q^{2}$ proposed by Busse [9] are given in Appendix A. Although the theory of Busse applies for a weak (linear) temperature dependency of the fluid properties and an infinite aspect of 
experimental cell, number of experimental investigations [10-16] confirm qualitatively some of the theoretical predictions. Differences between theory and experiments in the transition between hexagons and rolls arise as a consequence on one hand of a finite aspect ratio of the experimental system and on the other hand, away from the onset of convection the non-Boussinesq effects become stronger. A finite aspect ratio modifies the critical conditions [17], the wavenumber selection and the dynamics of hexagons rolls transition [16]. When the non-Boussinesq effects become stronger, the stability range of hexagons extends further in the strongly nonlinear regime $[11,18]$.

Another bifurcation sequence hexagons $\rightarrow$ rolls $\rightarrow$ hexagons was observed experimentally by Roy and Steinberg [19] using sulfur hexafluoride $\left(\mathrm{SF}_{6}\right)$ near the thermodynamic critical point. The restabilization of hexagons was termed 'reentrance'. The phenomenon of reentrant hexagons was predicted theoretically by Madruga, Riecke and Pesch [18], using an extension of the weakly nonlinear analysis of Busse [9]. However, this theoretical work predicts a smaller wavenumber than that observed experimentally.

Compared with the Newtonian fluids, very few experimental studies were devoted to non-Newtonian fluids. Here, we focus on shear-thinning fluids, i.e. fluids for which the viscosity decreases non linearly with the shear rate. This feature, when it is sufficiently strong leads to a subcritical bifurcation [20], [21],[22], [23].

The first experimental investigation of convection in a shear-thinning fluid confined between two horizontal plates was carried out by Pierre \& Tien [24]. The experimental cell is cylindrical with an aspect ratio $L=2$ where $L$ is the ratio of radius of the cylinder to the height of the fluid layer. The fluids used are aqueous solutions of Methocel (1wt \%) and Carbopol 934 (0.5, 0.75 and 1wt\%). The rheological behavior of these fluids was described by a power-law model, with a shear-thinning index ranging between 0.4 and 1 . The results were presented in terms of a correlation relating the Nusselt number $N u$ to Rayleigh and Prandtl numbers, for $10^{5} \leq R a \leq 10^{6}$. Later on, Tsiei \& Tien [25] extended this correlation to a wider range, $10^{3} \leq R a \leq 10^{6}$. For power-law fluids, Rayleigh and Prandtl numbers are defined with a viscosity calculated at a characteristic shear rate, which is the inverse of the thermal diffusion time. At the same period, Liang \& Acrivos [26] conducted an experimental study of the buoyancy-driven convection in horizontal layers of dilute aqueous solutions of polyacrylamide (Separan AP 30 at 0.5 and 1 wt 1\%). These fluids are shear-thinning with 
approximately constant viscosity at low shear-rate. The experimental setup consists of a rectangular cavity with the length to the height aspect ratio $L \approx 25$. The temperature difference between the top and bottom walls was kept smaller than $10^{\circ} \mathrm{C}$ to minimize the Boussinesq effects. Liang \& Acrivos [26] found that the critical Rayleigh number is the same as for a Newtonian fluid and that the shear-thinning behavior tends to increase the heat transfer.

Since Liang \& Acrivos in the early of 70's, there have been no experimental studies until 2016, where Darbouli et al. [27] investigated experimentally, using the MRI technique, the Rayleigh-Bénard convection for shear-thinning fluids. The experimental cell is cylindrical with an aspect ratio $L=3$. The fluids used are aqueous solutions of xanthan gum at different concentrations. These fluids are shear-thinning, weakly elastic and their viscosity varies weakly with the temperature. At low concentration of xanthan gum, the temperature difference at the onset of convection is small, the departure from Boussinesq approximations is weak and the convection takes place in the form of rolls for all the range of $R a$ examined $(1750<R a<3500)$. Note that Bouteraa et al. [28] have demonstrated in the framework of Boussinesq approximations, that near the threshold of convection, only rolls are stable and their stability is reinforced by the shear-thinning behavior. By increasing the concentration of xanthan gum, the zero shear viscosity increases and therefore a larger temperature difference is needed to start the convection. The departure from Boussinesq approximations becomes more significant and hexagons with upward motion in the centre are selected past the onset of convection. For larger Rayleigh number, hexagons become unstable with respect to rolls. For the highest concentration of xanthan gum, only hexagons are observed in all the range of $R a$ considered. However, in the paper of Darbouli et al. [27], the evolution of the wavenumber of the convective motions, that is of prime interest for the nonlinear theory of instabilities, has not been addressed. Furthermore, the hexagonal patterns are not clearly identified and the deviations from Boussinesq approximations have not been quantified.

From theoretical point of view, using a weakly nonlinear analysis, Vare et al. [29] demonstrated in the case of a a shear-thinning fluid with a thermodependent viscosity that the band of stable wavenumbers of hexagons is open and decentered to the right, i.e. to 
wavenumbers larger than the critical one. It thus appears that for sufficiently large Rayleigh number (depending on the viscosity contrast between the top and bottom), only wavenumbers larger than the critical one are allowed. To our best knowledge, there is no experimental data about the variation of the size of the hexagonal convection cells in Rayleigh-Bénard convection, when the temperature difference is increased above critical.

The objective of the present work is to provide an experimental support of the theoretical analysis of Vare et al. [29] that we have extended here to take into account the variation of the thermal expansion coefficient with temperature. We will show experimentally that the size of the hexagons decreases with increasing the temperature difference. Physical mechanisms underlying this variation will be discussed. Besides this, additional experimental data dealing with Rayleigh-Bénard convection in non-Newtonian fluids are provided. In particular, we show the possibility of obtaining what might be called "re-entrant" hexagons.

This paper is organized as follows. In Sec. 2, an extension of the theoretical analysis of Vare et al. [29] taking into account the temperature-dependence of the thermal expansion coefficient which plays a significant role in the experimental work is given. In Sec. 3 we describe the experimental set-up, the visualization method used, the rheological behavior of the fluids used and the dependence of their properties on temperature. In Sec. 4, the experimental results are presented and discussed mainly focusing on the evolution of the wavenumber. Finally, conclusions are presented in Sec. 5 .

\section{THEORETICAL TOOLS}

We consider a layer of a shear-thinning fluid of thickness $\hat{d}$ confined between two impermeable horizontal plates, infinite in extent, which are perfect heat conductors. The fluid is

heated from below at temperature $\hat{T}_{1}=\hat{T}_{0}+\Delta \hat{T} / 2$ and cooled from above at temperature $\hat{T}_{2}=\hat{T}_{0}-\Delta \hat{T} / 2$ with $\Delta \hat{T}>0$. The fluid has a thermal diffusivity $\hat{\kappa}$ and viscosity $\hat{\mu}_{0}$ at zero-shear-rate. As in [9], the temperature dependency of the density

$$
\hat{\rho}=\hat{\rho}_{0}\left[1-\hat{\chi}\left(\hat{T}-\hat{T}_{0}\right)\right] \quad \text { with } \quad \hat{\chi}=\hat{\alpha}+\hat{\beta}\left(\hat{T}-\hat{T}_{0}\right)
$$


will be taken into account only in the gravity term of the momentum equations. In the absence of convection, the heat conducting state is described by

$$
\hat{\boldsymbol{u}}=0 \quad \text { and } \quad \hat{T}_{c}-\hat{T}_{0}=\frac{\Delta \hat{T}}{2}\left(1-\frac{2 \hat{z}}{\hat{d}}\right),
$$

where $\hat{\boldsymbol{u}}$ is the fluid velocity and $\hat{T}_{c}$ the conductive temperature profile. The $z$-axis is directed upwards with its origin located at the bottom plate. Using the units $\hat{d}^{2} / \hat{\kappa}, \hat{d}, \hat{\kappa} / \hat{d}$ and $\Delta \hat{T}$ for time, length, velocity and temperature, the dimensionless perturbation equations are:

$$
\begin{aligned}
& \boldsymbol{\nabla} \cdot \boldsymbol{u}=0 \\
& \frac{1}{\operatorname{Pr}}\left[\frac{\partial \boldsymbol{u}}{\partial t}+(\boldsymbol{u} \cdot \boldsymbol{\nabla}) \boldsymbol{u}\right]=-\boldsymbol{\nabla} p+R a \theta \boldsymbol{e}_{z}+2 R a \gamma_{1}\left(T_{c}-T_{0}\right) \theta \boldsymbol{e}_{z}+ \\
& R a \gamma_{1} \theta^{2} \boldsymbol{e}_{z}+\nabla \cdot \boldsymbol{\tau} \\
& \frac{\partial \theta}{\partial t}+\boldsymbol{u} \cdot \nabla \theta=\boldsymbol{u} \cdot \boldsymbol{e}_{z}+\nabla^{2} \theta
\end{aligned}
$$

where

$$
\gamma_{1}=\frac{\hat{\beta} \Delta \hat{T}}{\hat{\alpha}} .
$$

Here, $\boldsymbol{e}_{z}$ denotes the unit vector in the vertical direction, $p(\boldsymbol{x}, t)$ and $\theta(\boldsymbol{x}, t)$ represent the pressure and temperature deviations from their values in the conductive state. The quantities defined with a hat (^) are dimensional, while quantities without (^) are dimensionless. The Rayleigh number, $R a$, and the Prandtl number $\operatorname{Pr}$ are

$$
R a=\frac{\hat{\alpha} \hat{\rho}_{0} \hat{g} \Delta \hat{T} \hat{d}^{3}}{\hat{\kappa} \hat{\overline{\mu_{0}}}} \quad ; \quad \operatorname{Pr}=\frac{\hat{\overline{\mu_{0}}}}{\hat{\rho}_{0} \hat{\kappa}}
$$

The reference viscosity $\hat{\bar{\mu}}_{0}$ is the zero-shear-rate viscosity evaluated at $\hat{T}_{0}$. The fluid is assumed to be purely viscous and shear-thinning. The non-dimensionalized deviatoric stresstensor $\boldsymbol{\tau}$ is related to the strain-rate tensor:

$$
\dot{\gamma}=\boldsymbol{\nabla} \boldsymbol{u}+(\boldsymbol{\nabla} \boldsymbol{u})^{T}
$$

by the relation

$$
\boldsymbol{\tau}=\mu(\Gamma) \dot{\gamma} \quad \text { where } \quad \Gamma=\frac{1}{2} \dot{\gamma}_{i j} \dot{\gamma}_{i j}
$$

is the second invariant of the strain-rate tensor. The viscosity $\mu$ is modeled by the Carreau model and we assume that $\mu$ depends exponentially on temperature:

$$
\frac{\mu-\mu_{\infty}}{\mu_{0}-\mu_{\infty}}=\exp \left[-\hat{b}\left(\hat{T}-\hat{T}_{0}\right)\right]\left(1+\hat{\lambda}^{2} \hat{\Gamma}\right)^{\left(n_{c}-1\right) / 2},
$$


where $\mu_{0}=\hat{\mu}_{0} / \hat{\bar{\mu}}_{0}$ and $\mu_{\infty}=\hat{\mu}_{\infty} / \hat{\bar{\mu}}_{0}$ are the dimensionless dynamic viscosity at low and high shear rate, $n_{c}<1$, the shear-thinning index and $\hat{\lambda}$ the characteristic time of the fluid. The charecteristic shear-rate for the transition from the Newtonian plateau to the shear-thinning regime is determined by $1 / \hat{\lambda}$. The infinite shear-viscosity $\mu_{\infty}$ is generally associated with the breakdown of the fluid and is frequently significantly smaller, $\left(10^{3}-10^{4}\right)$ times smaller than $\mu_{0}$ see Bird et al. [30]. This leaves three rheological parameters $\mu_{0}, \lambda$ and $n_{c}$ and the nondimensional viscosity in (13) writes

$$
\frac{\mu}{\mu_{0}}=\mu_{b}(z) \exp (-c \theta)\left(1+\lambda^{2} \Gamma\right)^{\left(n_{c}-1\right) / 2},
$$

where $\mu_{b}(z)=\exp (c(z-1 / 2))$ is the viscosity profile at quiescent state, $c=\hat{b} \Delta \hat{T}$ a measure of the viscosity contrast and $\lambda=\hat{\lambda} \hat{d}^{2} / \hat{\kappa}_{0}$ a dimensionless characteristic time of the fluid. The viscosity ratio across the fluid layer is given by

$$
r=\frac{\mu_{b}(z=1)}{\mu_{b}(z=0)}=\exp (c) .
$$

For a small amplitude disturbance, the viscosity can be expanded about the hydrostatic solution

$$
\mu=\mu_{b}(z)[1-c \theta+\ldots]\left[1+\left(\frac{n_{c}-1}{2}\right) \lambda^{2} \Gamma+\ldots\right]
$$

At nonlinear order Taylor expansion of $\left(1+\lambda^{2} \Gamma\right)^{\frac{n_{c}-1}{2}}$, a relevant rheological parameter appears, i.e. the degree of shear-thinning at the onset of convection:

$$
S_{t h}=\frac{1-n_{c}}{2} \lambda^{2}=\left|\frac{\partial \mu}{\partial \Gamma}\right|_{\Gamma=0, z=1 / 2} .
$$

Equations (6) - (8), (12) and (14) have to be completed by appropriate boundary conditions. For the velocity field, no-slip boundary conditions are considered. For the temperature deviation, the thermal conductivity of the boundaries is assumed much larger than that of the fluid, so that their temperature remains fixed. The boundary conditions are then

$$
u=v=w=\theta=0 \quad \text { on } \quad z=0,1 .
$$

\section{A. Linear stability analysis: critical Rayleigh number and wavenumber}

In the linear theory $\boldsymbol{u}$ and $\theta$ are assumed infinitesimal and the nonlinear terms in the perturbation equations are neglected." At this stage, non-Newtonian effects do not come 


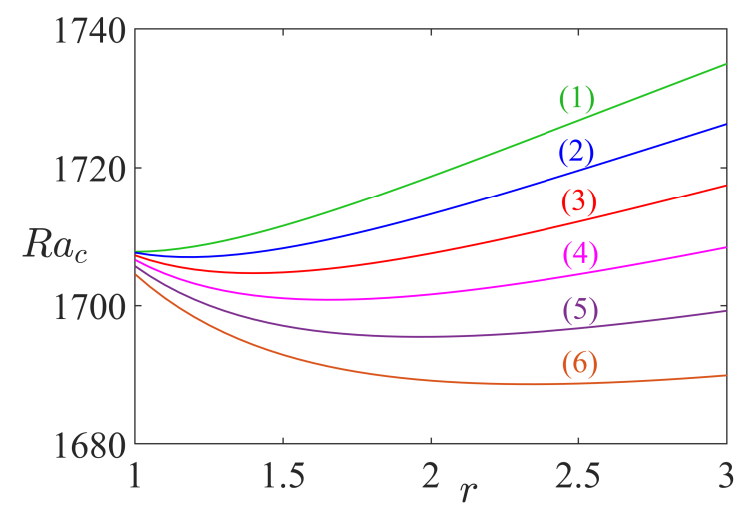

(a)

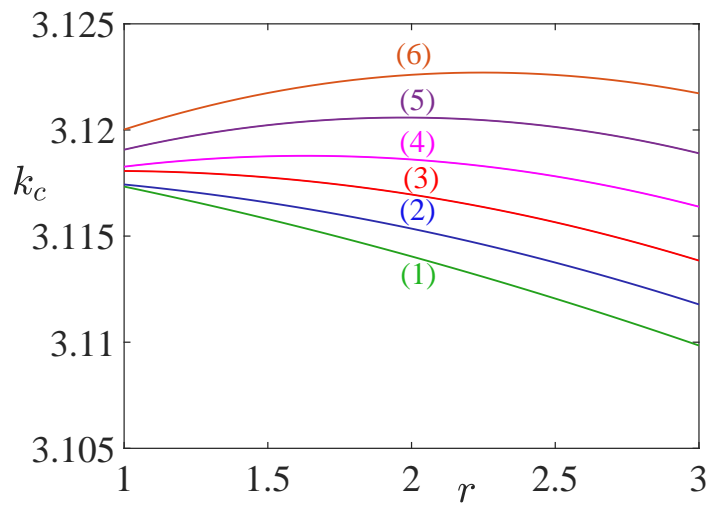

(b)

FIG. 1. Variation of the critical Rayleigh number (a) and the critical wavenumber (b) as a function of the viscosity ratio for different values of the parameter $\gamma_{1}$ defined in equation (9). (1) $\gamma_{1}=0$, (2) $\gamma_{1}=0.1,(3) \gamma_{1}=0.2$, (4) $\gamma_{1}=0.3,(5) \gamma_{1}=0.4,(6) \gamma_{1}=0.5$.

into play". We seek a normal mode solution

$$
(u, v, w, \theta)=\left(U_{11}, V_{11}, W_{11}, G_{11}\right) f(x, y) \exp (\sigma t)
$$

where $f(x, y)=\exp \left(i k_{x} x+i k_{y} y\right)$ is the planform function, $\boldsymbol{k}=\left(k_{x}, k_{y}, 0\right)$ the horizontal wave vector and $\sigma=\sigma_{r}+i \sigma_{i}$ a complex number. Substituting (19) into the linearized perturbation equations, lead to an eigenvalue problem which is solved by a Chebyshev collocation method. It is easy to show that all eigenvalues of the linearized problem are real $\left(\sigma_{i}=0\right)$, i.e. the convection occurring initially is stationary. By setting $\sigma_{r}=0$, one obtains the marginal stability curve $R a(k)$, where $k$ is the norm of the eigenvector $\boldsymbol{k}$. The minimum of $R a(k)$ gives the critical Rayleigh number $R a_{c}$ and the corresponding wavenumber $k_{c}$. The critical conditions $R a_{c}$ and $k_{c}$ are the same as those for a Newtonian fluid. Figure 1 displays the variation of the critical number $R a_{c}$ for the onset of convection as well $k_{c}$ the critical wavenumber as a function of the viscosity ratio $r$ for different values of the parameter $\gamma_{1}$. For an exponential law of the viscosity as a function of temperature and for the range of the parameters considered, it is observed that $R a_{c}$ increases with increasing the viscosity ratio between the top and the bottom walls. This is accompanied by a very weak decrease of the critical wavenumber as the viscosity ratio increases. In contrast, an increase of $\gamma_{1}$ tends to reduce $R a_{c}$ and increases slightly $k_{c}$. 


\section{B. Weakly nonlinear analysis}

As it is known, linear stability analysis gives the critical Rayleigh number for instability onset and determines the modulus $k_{c}$ of the critical wave vector $\boldsymbol{k}$. However, the direction of $\boldsymbol{k}$ is arbitrary because of the isotropy of the extended horizontal plane. In addition any linear combination of normal modes $A_{p} \exp \left(i \boldsymbol{k}_{p} \cdot \boldsymbol{r}\right)\left(U_{11}(z), V_{11}(z), W_{11}(z), G_{11}(z)\right)$ where $\boldsymbol{r}=(x, y), \boldsymbol{k}_{p}=\left(k_{p x}, k_{p y},\left|\boldsymbol{k}_{p}\right|=k_{c}\right)$ and $A_{p}$ are constant coefficients is a solution of the linear problem, i.e. there is also a pattern degeneracy. The pattern selection is determined by the nonlinear terms. A weakly nonlinear analysis can be adopted as a first approach to investigate nonlinear effects in the competition, existence and stability of rolls and hexagons. Hereafter, we consider the case where the wavevectors lie on a hexagonal lattice

$$
(u, v, w, \theta)=\sum_{p=1}^{p=3} A_{p}\left(U_{11}, V_{11}, W_{11}, G_{11}\right) \exp i \boldsymbol{k}_{p} \cdot \boldsymbol{r}+\text { c.c. }+ \text { h.o.t. },
$$

where "c.c." denotes the complex conjugate of the prior expression and "h.o.t." stands for higher order terms. The hexagon patterns are made of three pairs of wavevectors at $2 \pi / 3$ an-

gles apart: $\boldsymbol{k}_{1}=k_{c} \boldsymbol{e}_{x}, \boldsymbol{k}_{2}=k_{c}\left(-(1 / 2) \boldsymbol{e}_{x}+(\sqrt{3} / 2) \boldsymbol{e}_{y}\right)$ and $\boldsymbol{k}_{3}=k_{c}\left(-(1 / 2) \boldsymbol{e}_{x}-(\sqrt{3} / 2) \boldsymbol{e}_{y}\right)$. As the Rayleigh number is increased above the onset $R a_{c}$, the growth-rate of the perturbation is positive for any wavenumber within a band $\mathrm{O}(\sqrt{\epsilon})$ around the critical wavenumber. The effect of the small band of unstable wavenumbers around $k_{c}$, is to modulate the envelope of the carrier wave, $\exp \left(i \boldsymbol{k}_{p} \cdot \boldsymbol{r}\right)$, so that the amplitude $A_{p}$ varies slowly in time and space. The slow time variable $T=\epsilon t$ and the slow variables space $X=\sqrt{\epsilon} x$ and $Y=\sqrt{\epsilon} y$ are introduced. We then write

$$
(u, v, w, \theta)=\sum_{p=1}^{p=3} A_{p}(X, Y, T)\left(U_{11}, V_{11}, W_{11}, G_{11}\right) \exp \left(i \boldsymbol{k}_{p} \cdot \boldsymbol{r}\right)+\text { c.c. }+ \text { h.o.t } .
$$

A multiple scale perturbation theory developed in the vicinity of the onset allows to determine the evolution equation for $A_{p}(X, Y, T)$. Details can be found in [29] and references therein. Up to third order in perturbation equations, the spatio-temporal evolution of the amplitude $A_{p}$ is described through the equations

$$
\begin{aligned}
\frac{\partial A_{1}}{\partial t}= & \frac{\epsilon}{\tau_{0}} A_{1}+\frac{\xi_{0}^{2}}{\tau_{0}}\left(\boldsymbol{n}_{1} \cdot \boldsymbol{\nabla}_{H x}\right)^{2}+\zeta A_{2}^{*} A_{3}^{*}- \\
& g_{1}\left|A_{1}\right|^{2} A_{1}-g_{2}\left(\left|A_{2}\right|^{2}+\left|A_{3}\right|^{2}\right) A_{1}+ \\
& i \alpha_{1}\left[A_{2}^{*}\left(\boldsymbol{n}_{3} \cdot \boldsymbol{\nabla}_{H x}\right)+A_{3}^{*}\left(\boldsymbol{n}_{2} \cdot \boldsymbol{\nabla}_{H x}\right) A_{2}^{*}\right]+ \\
& i \alpha_{2}\left[A_{2}^{*}\left(\boldsymbol{\tau}_{3} \cdot \nabla_{H x}\right) A_{3}^{*}+A_{3}^{*}\left(\boldsymbol{\tau}_{2} \cdot \nabla_{H x}\right) A_{2}^{*}\right],
\end{aligned}
$$


where, $A_{i}^{*}$ is the complex conjugate of $A_{i}, \nabla_{H x}$ the horizontal gradient for the fast variables, $\tau_{0}$ the characteristic time for the instability to grow, $\xi_{0}$ the coherence length, $\zeta$ the coefficient of quadratic nonlinearity describing non-Boussinesq effects, $g_{1}$ refers to the self-saturation coefficient and $g_{2}$ coefficient of cubic interaction of rolls of different orientation. The terms with $\alpha_{1}$ account for distortions in the direction of rolls and therefore corresponds physically to wavenumber dilatation. The coefficient $\alpha_{1}$ is positive and increases with increasing $r$ and $\gamma_{1}$. The terms with $\alpha_{2}$ account for distortion in the hexagonal form. It is smaller than $\alpha_{1}$. The equations for $A_{2}, A_{3}$ are obtained by cyclic permutation of the indices. Here $\boldsymbol{n}_{i}$ is the unit vector oriented along the wavevector $\boldsymbol{k}_{i}$, and $\boldsymbol{\tau}_{i}$ orthogonal to $\boldsymbol{n}_{i}$. Coefficients of Eq. (22) have been calculated and correlations are proposed in Appendix B. Note that the the terms with $\alpha_{1}$ and $\alpha_{2}$ render the system (22) nonpotential [31].

The homogeneous and stationary solutions of equation (22), including slightly off-critical wavenumber in the amplitude $\left(A_{p}=\mathcal{A}_{p} \exp \left(i \boldsymbol{q}_{p} \cdot \boldsymbol{r}\right)\right)$ are: (a) rolls $\mathcal{A}_{1} \neq 0, \mathcal{A}_{2}=\mathcal{A}_{3}=0$, hexagons with $\mathcal{A}_{1}=\mathcal{A}_{2}=\mathcal{A}_{3}$ and mixed states with $\mathcal{A}_{1} \neq 0, \mathcal{A}_{2}=\mathcal{A}_{3} \neq 0$. A linear stability analysis of these stationary and homogeneous solutions with respect to homogeneous perturbations shows that the mixed state is always unstable, the hexagons are stable in the range $\epsilon_{a} \leq \epsilon \leq \epsilon_{h}$, while the rolls are stable when $g_{2}>g_{1}$ and $\epsilon \geq \epsilon_{r}$, where the transition thresholds $\epsilon_{a}, \epsilon_{h}, \epsilon_{r}$ are given by:

$$
\begin{aligned}
\epsilon_{a} & =-\frac{\left(\zeta+2 \alpha_{1} q\right)^{2} \tau_{0}}{4\left(g_{1}+2 g_{2}\right)}+\xi_{0}^{2} q^{2}, \\
\epsilon_{h} & =\frac{\tau_{0}\left(\zeta+2 \alpha_{1} q\right)^{2}\left(2 g_{1}+g_{2}\right)}{\left(g_{1}-g_{2}\right)^{2}}+\xi_{0}^{2} q^{2}, \\
\epsilon_{r} & =\frac{\tau_{0} g_{1}\left(\zeta+2 \alpha_{1} q\right)^{2}}{\left(g_{2}-g_{1}\right)^{2}}+\xi_{0}^{2} q^{2}
\end{aligned}
$$

An example of the bifurcation diagram is shown in figure 2. It is obtained for a Carreau fluid with a shear-thinning degree $S_{t h}=5 \times 10^{-5}, k=k_{c}(q=0), \gamma_{1}=0.2$ and a viscosity ratio $r=2$. Hexagons bifurcate transcritically from the conductive state where they are unstable. Both hexagons and the conductive state are stable in the range $\epsilon_{a} \leq \epsilon \leq 0$ and both hexagons and rolls are stable in the range $\epsilon_{r} \leq \epsilon \leq \epsilon_{h}$. In this range, rolls and hexagons solutions are linked via a branch of mixed modes.

Variations of $\epsilon_{a}, \epsilon_{r}$ and $\epsilon_{h}$ as a function of the viscosities ratios $r$ for different values of the shear-thinning degree $S_{t h}$ and two values of the parameter $\gamma_{1}$ are depicted in figure 3 . Overall, 


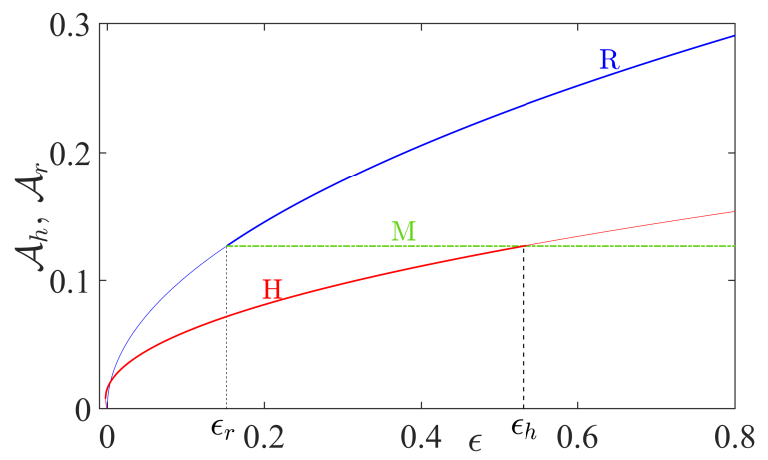

FIG. 2. Bifurcation diagram for hexagons in the case where $g_{2}>g_{1}$ with the parameters $r=2, q=$ $0\left(k=k_{c}\right), \gamma_{1}=0.2$ and $S_{t h}=5 \times 10^{-5}$. The amplitude $\left|A_{1}\right|$ is plotted against the distance to the threshold $\epsilon$, for the roll-solution (labelled R), for the mixed mode branch (labelled M) and for the hexagon solution labelled $(\mathrm{H})$. Thick lines indicate stable solutions and thin lines represent unstable solutions.

the thermodependency of the viscosity and the parameter $\gamma_{1}$ (equation 9) favor convection in form of hexagons and their stability whereas shear-thinning effects favor convection in form of rolls and their stability. In other words, the domain of stability of hexagons increase with increasing $r$ and $\gamma_{1}$ and decreases with increasing shear-thinning effects. One can also note in figures 3(a) and 3(b) that $\left|\epsilon_{a}\right|$ increases with increasing $S_{t h}$ as shear-thinning effects favor a subcritical bifurcation [28]. The correlations proposed by Busse [9] for a Newtonian fluid assuming a linear variation of the viscosity with temperature (see Appendix E) are displayed for comparison. As it can be observed, the difference between the linear and the exponential models increases with increasing $r$. Another kind of destabilizing perturbations are those involving spatial modulations over distances much larger than the basic wavelength. The amplitude of a slightly distorted hexagons is written as

$$
A_{p}=\left(\mathcal{A} p+r_{p}\right) \exp \left[i\left(q x_{p}+\phi_{p}\right)\right], p=1,2,3
$$

where $x_{p}=\boldsymbol{n}_{p} \cdot \boldsymbol{r},\left|r_{p}\left(x_{1}, x_{2}, x_{3}, t\right)\right|<<1$ is the amplitude of the perturbation, and $\left|\phi_{p}\left(x_{1}, x_{2}, x_{3}, t\right)\right|<<1$ is the phase of the perturbation respectively. Substituting $A_{p}$ by its expression 26 in the amplitude equations leads after linearization to six equations in $r_{p}$ and $\phi_{p}$. In the case of long wave length limit of the perturbation, it is shown [31-34] that the amplitudes $r_{p}$ and the total phase $\Phi=\phi_{1}+\phi_{2}+\phi_{3}$ are fastly relaxing and therefore can be eliminated adiabatically. That leaves $\phi_{2}$ and $\phi_{3}$ which are used to express a vector phase 


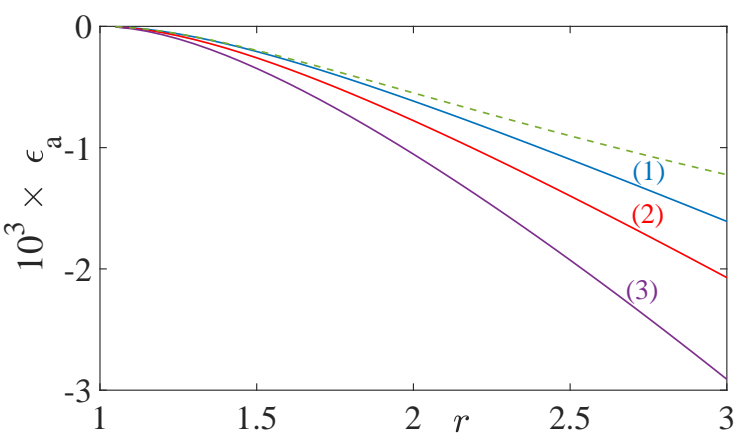

(a)

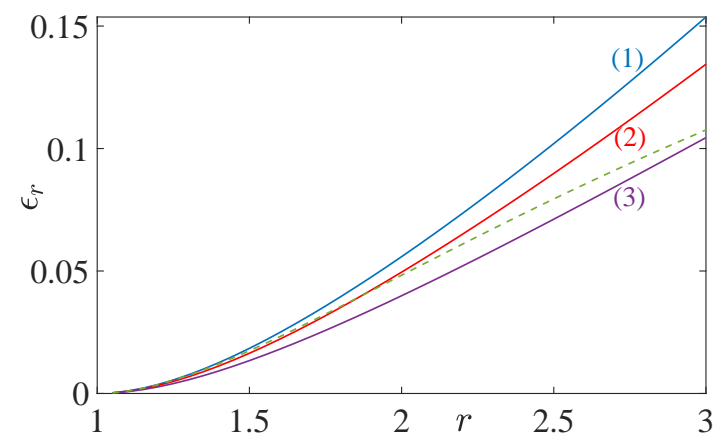

(c)

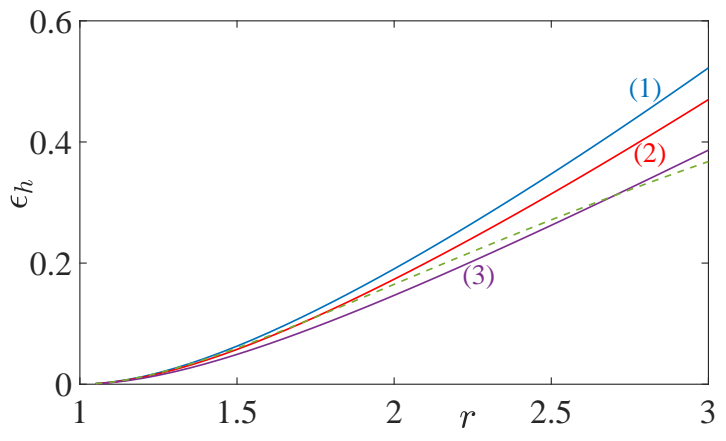

(e)

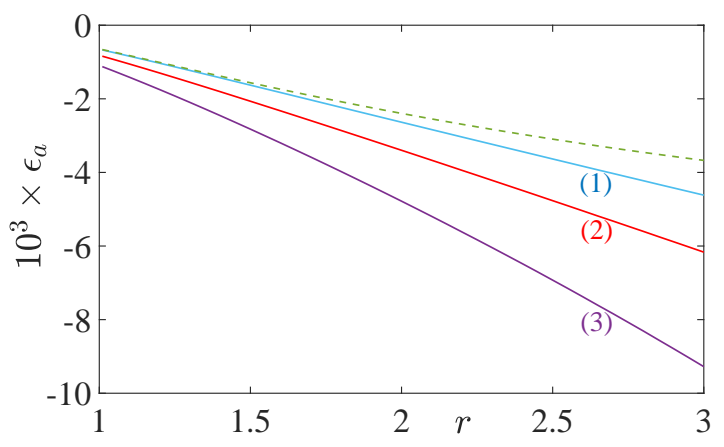

(b)

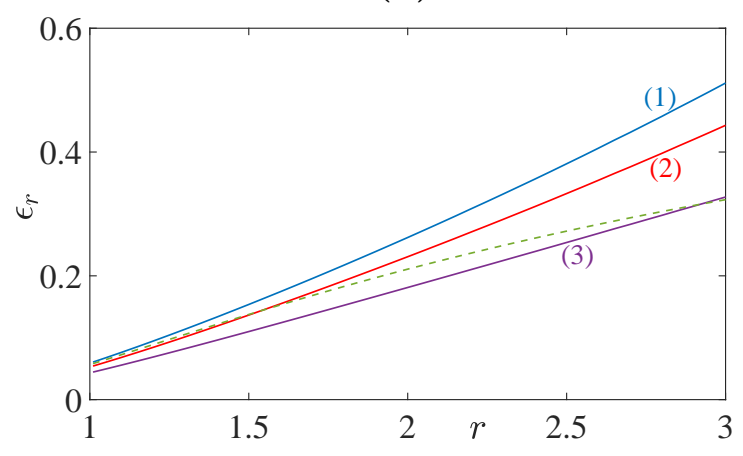

(d)

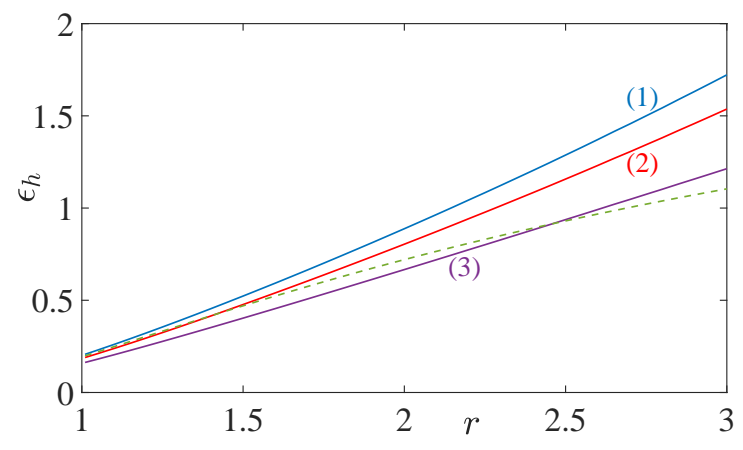

(f)

FIG. 3. Variations of $\epsilon_{a}, \epsilon_{r}$ and $\epsilon_{h}$ as a function of $r$ for three values of the shear-thinning degree $S_{t h}$. The Prandtl number is fixed $P r=100$. (a) $\gamma_{1}=0$, (b) $\gamma_{1}=0.3$, (c) $\gamma_{1}=0$, (d) $\gamma_{1}=0.3$, (e) $\gamma_{1}=0$, (f) $\gamma_{1}=0.3$. Curve (1) $S_{t h}=0$, i.e. Newtonian fluid; curve (2) $S_{t h}=5 \times 10^{-5}$ and curve (3) $S_{t h}=10^{-4}$. The dashed line is the correlation proposed by Busse [9].

$\boldsymbol{\Phi}=\left[-\left(\phi_{2}+\phi_{3}\right),\left(\phi_{2}-\phi_{3}\right) / \sqrt{3}\right]$. The following phase diffusion equation is then obtained:

$$
\frac{\partial \boldsymbol{\Phi}}{\partial t}=D_{\perp} \boldsymbol{\nabla}^{2} \boldsymbol{\Phi}+\left(D_{\|}-D_{\perp}\right) \boldsymbol{\nabla}(\boldsymbol{\nabla} \cdot \boldsymbol{\Phi}),
$$

where $D_{\perp}$ and $D_{\|}$are the transverse and longitudinal diffusion equations. Their expressions 


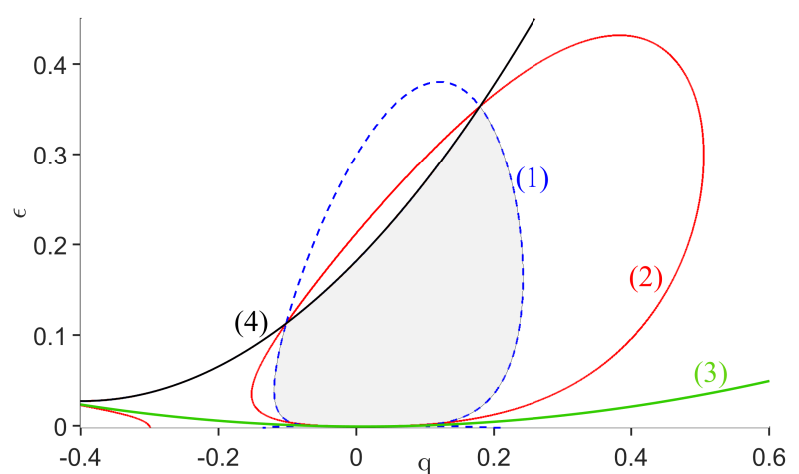

(a)

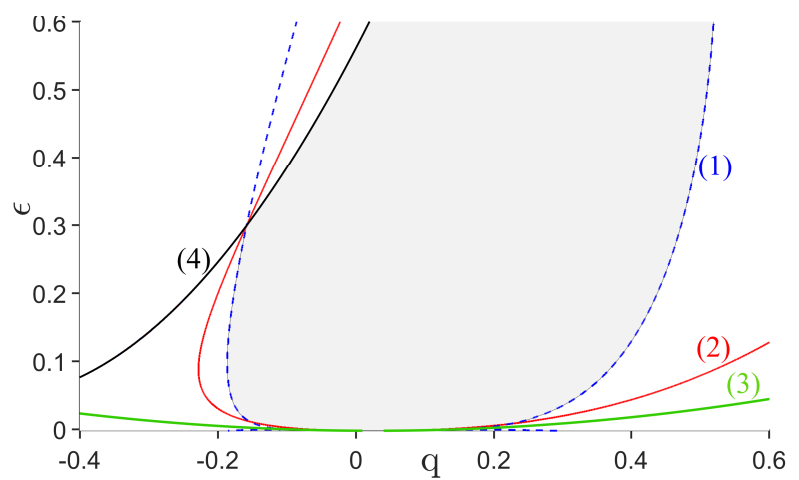

(b)

FIG. 4. Hexagon stability diagram for a shear-thinning fluid with $S_{t h}=5 \times 10^{-5}$ at $\operatorname{Pr}=100$ and two different values of the parameter $\gamma_{1}$. The viscosity ratio is fixed, $r=2$. (a) $\gamma_{1}=0$, (b) $\gamma_{1}=0.2$. Curve (1) $D_{\perp}=0$, curve (2) $D_{\|}=0$, curve (3) bifurcation from the conductive state to convection with hexagons, curve (4) bifurcation from hexagons to rolls.

as a function of $\xi_{0}, \tau_{0}, \alpha_{1}, \alpha_{2}, g_{1}$ and $g_{2}$ are given in Appendix C. Following [31-35], the phase $\boldsymbol{\Phi}$ can be split into a transverse part $\boldsymbol{\Phi}_{t}$ that satisfies $\boldsymbol{\nabla} \cdot \boldsymbol{\Phi}_{t}=0$ and a longitudinal part that satisfies $\boldsymbol{\nabla} \times \boldsymbol{\Phi}_{\ell}=0$. This leads to the uncoupled phase diffusion equations:

$$
\frac{\partial \boldsymbol{\Phi}_{\ell}}{\partial t}=D_{\|} \boldsymbol{\Phi}_{\ell} \quad, \quad \frac{\partial \boldsymbol{\Phi}_{t}}{\partial t}=D_{\perp} \boldsymbol{\Phi}_{t}
$$

The hexagonal patterns are stable to phase perturbations provided that $D_{\perp}>0$ and $D_{\|}>0$. In figure 4 we show the phase stability diagrams for two values of the parameter $\gamma_{1}$. The curves $D_{\perp}=0$ and $D_{\|}=0$ determine the boundary instability to transverse and longitudinal phase perturbations respectively. We have also included the amplitude stability curves $\epsilon_{h}$ (curve 4) and $\epsilon_{a}$ (curve 3). Hexagons are stable in the shaded region. For Carreau fluid with $S_{t h}=5 \times 10^{-5}, r=2$ and $\gamma_{1}=0$, the region of stability to amplitude and phase modes is closed and bent to the right mainly owing to the positive sign of $\alpha_{1}$. With increasing the parameter $\gamma_{1}$, the stability domain of hexagons increases significantly and it is more decentered towards the right. For the two cases, the domain of hexagons stability is mainly delimited by the amplitude stability curves and the transverse phase instability boundary.

In the domain where the hexagons are stable, the variation of their amplitude as a function of the shear-thinning degree and the thermodependency of the fluid properties can be illustrated through the convective heat transport. The Nusselt number, $N u$, the ratio of the total heat flux to the purely conductive heat flux in the absence of fluid flow can be 


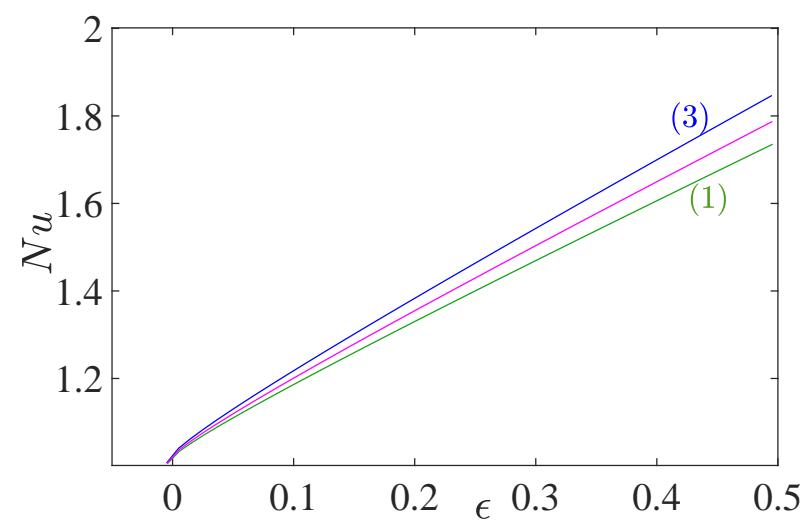

FIG. 5. Variation of Nusselt as a function of the distance to the onset of convection $\epsilon$ for Newtonian and Carreau fluids with $r=2.5$ and $\gamma_{1}=0.36$. (1) $\alpha=0$ (Newtonian fluid), (2) $\alpha=1.25 \times 10^{-5}$, (3) $\alpha=2.5 \times 10^{-5}$.

calculated at the lower plate, as

$$
N u=1-\left(\frac{\partial \bar{\theta}}{\partial z}\right)_{z=0}=1-\left(\frac{d T_{1}(z)}{d z}\right)_{z=0}\left(\left|A_{1}\right|^{2}+\left|A_{2}\right|^{2}+\left|A_{3}\right|^{2}\right),
$$

where the overbar denotes the horizontal average over one wavelength and $T_{1}(z)$ is the correction at the second order of the conductive temperature profile. It arises from the interaction of the fundamental mode with its complex conjugate. Note that $\left(d T_{1} / d z\right)_{z=0}<0$. In the case of homogeneous hexagons, with $k=k_{c}$, the amplitude is given by

$$
\left|A_{1}\right|=\left|A_{2}\right|=\left|A_{3}\right|=\frac{\zeta+\sqrt{\zeta^{2}+4 \epsilon\left(g_{1}+2 g_{2}\right) / \tau_{0}}}{2\left(g_{1}+2 g_{2}\right)} .
$$

Figure 5 shows the variation of the Nusselt number as a function of the distance to the onset of convection, $\epsilon$, for Newtonian and Carreau fluids with fixed $r$ and $\gamma_{1}$. As expected, the Nusselt number increases with increasing shear-thinning effects.

Concerning the influence of non-Boussinesq effects on heat transfer, it is illustrated in figure 6. We have represented $N u$ as a function of $\epsilon$ either for increasing the viscosity ratio (figure $6(\mathrm{a})$ ) or increasing $\gamma_{1}$ (figure 6(b)). The heat transfer is enhanced by non-Boussinesq effects. Actually, $\zeta$ in equation (30) is a coefficient describing non-Boussinesq effects. For moderate departures from Boussinesq approximation (say $|Q| \leq 5$ ) and for $\operatorname{Pr} \geq 100, \zeta$ can be related to the parameter $Q$ introduced by Busse [9] by the following correlation:

$$
\zeta=3.57|Q|
$$




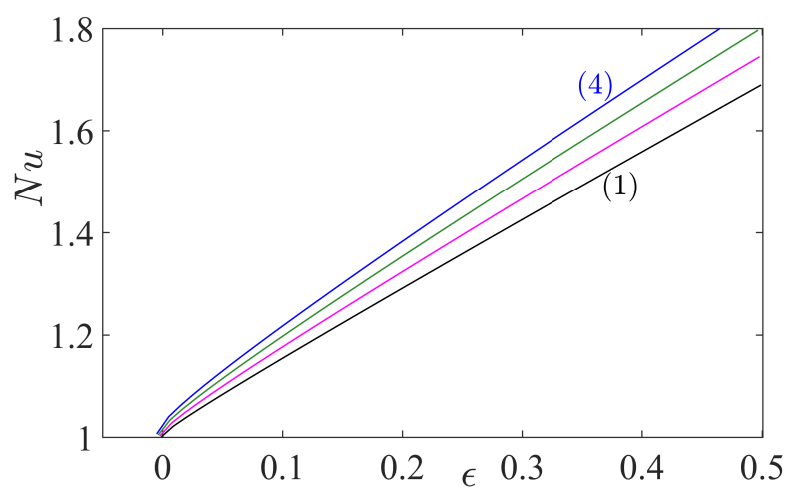

(a)

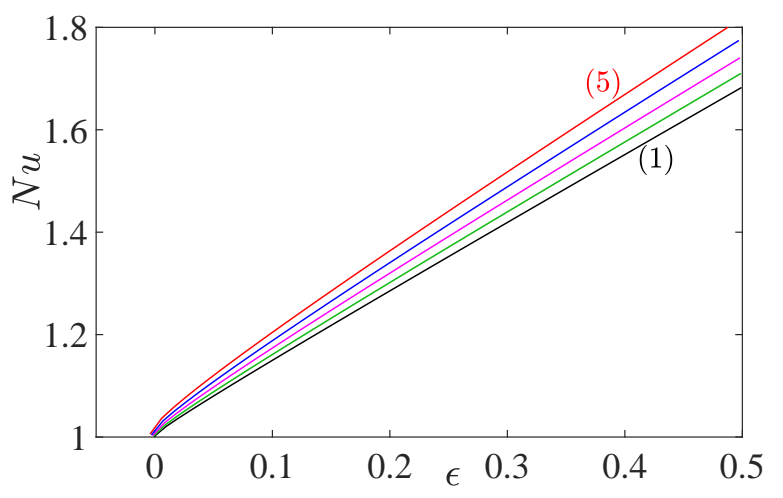

(b)

FIG. 6. Variation of Nusselt number as a function of the distance to the onset of convection $\epsilon$ for a Carreau fluid with $S_{t h}=5 \times 10^{-5}$ and $\operatorname{Pr}=100$. (a) $\gamma_{1}=0.36$, (1) $r=1$, (2) $r=1.5$, (3) $r=2$ and (4) $r=2.5$. (b) $r=2$, (1) $\gamma_{1}=0$, (2) $\gamma_{1}=0.1$, (3) $\gamma_{1}=0.2$, (4) $\gamma_{1}=0.3$ and (5) $\gamma_{1}=0.4$.

Equations (29)-(31) show that near the onset, the deviation of $N u$ from the Boussinesq value is of order $Q^{2}$. The heat transfer enhancement is related to the increase in convection amplitude with the strength of non-Boussinesq effects. Solomatov \& Jain [36] have shown numerically that this trend remains valid even for viscosity contrasts up to $10^{10}$ which can occur in the interior of planets.

\section{APPARATUS, FLUIDS USED AND EXPERIMENTAL PROCEDURE}

\section{A. Experimental set-up}

The fluid is confined in a cylindrical container of radius $\hat{R}=60 \mathrm{~mm}$ and height adjustable at $\hat{d}=15 \mathrm{~mm}$ and $\hat{d}=20 \mathrm{~mm}$. The aspect ratio $L=\hat{R} / \hat{d}$ is 4 and 3 respectively. The lateral walls are made of polymethyl methacrylate (PMMA) of $10 \mathrm{~mm}$ thick which is an insulated thermoplastic material. To allow shadowgraph visualizations of the thermoconvective pattern, the top and bottom horizontal plates are transparent. The plates used of thickness $3 \mathrm{~mm}$ are made of sapphire. This material has excellent optical properties and a large thermal conductivity, $\hat{k}_{p}=35 \mathrm{~W} \cdot \mathrm{m}^{-1} \cdot \mathrm{K}^{-1}$, as compared to that of the fluids used. The top and bottom plates are surrounded by a cylindrical chamber, made of PMMA, containing

circulating water at temperature $\hat{T}_{t}$ and $\hat{T}_{b}$ respectively. The schematic of the experimental setup is displayed in figure 7 . The circulating water is provided by two thermostatic baths. 
TABLE I. Characteristics of the optical arrangement

\begin{tabular}{lc}
\hline \hline Focal length of the focusing lens $(\mathrm{mm})$ & 400 \\
Lens diameter $(\mathrm{mm})$ & 200 \\
pinhole diameter $(\mathrm{mm})$ & 0.3 \\
Camera model & Lw115M \\
Camera Lens: Focal range $(\mathrm{mm})$ & $13-130$ \\
$\quad$ : Iris & F5.6-F32C \\
\hline \hline
\end{tabular}

The water bath temperatures are set constant to within $\pm 0.01^{\circ} \mathrm{C}$. The temperatures of the top and bottom plates are measured with Pt-100 sensors potted at outlet and inlet of circulating water. Heat losses are limited by fixing the mean temperature of the fluid layer to $\hat{T}_{0}=24^{\circ} \mathrm{C}$, which corresponds to the average temperature of the handling room. It can be shown straightforwardly that more than 99 percent of the measured temperature difference $\hat{T}_{b}-\hat{T}_{t}$ was across the fluid layer, $\Delta \hat{T}$. In order to avoid possible consequences of time dependence of the variation of temperature, $\Delta \hat{T}$ was varied extremely slowly. The temperature difference, across the fluid layer was varied by step of $0.1^{\circ} \mathrm{C}$ and was held at each step during 180 minutes. Hence, during one day, the increase of $\Delta \hat{T}$ was of $0.8^{\circ} \mathrm{C}$. That means that the experiments had to run continously for a couple of weeks in order to reach the maximal temperature difference required. Note that the vertical diffusion time $\hat{t}_{v} \equiv \hat{d}^{2} / \hat{\kappa} \approx 28$ and 50 minutes for $\hat{d}=15$ and $20 \mathrm{~mm}$ respectively $(\hat{\kappa}$ is the thermal diffusivity of the fluid used).

Observation of the convective patterns is realized by using standard shadowgraph technique (figure 8). This technique relies upon the variation of the refractive index of a fluid with its density and hence its temperature. A parallel light beam crossing the fluid layer is focused where the the refractive index is large (cold stream) and diverges where the refractive index is low (warm stream). The modulation of the light intensity reveals the structure of the convection pattern. In order to avoid chromatic aberrations in the optical system and the fluid layer and to avoid also the production of interference fringes that obscure the shadowgraph effect, the light source should be monochromatic and incoherent. Therefore Light Emitting Diode LED was found to be a good compromise. To get a pseudo-point source, a $0.3 \mathrm{~mm}$ diameter pinhole was glued above the LED surface. This technique is 


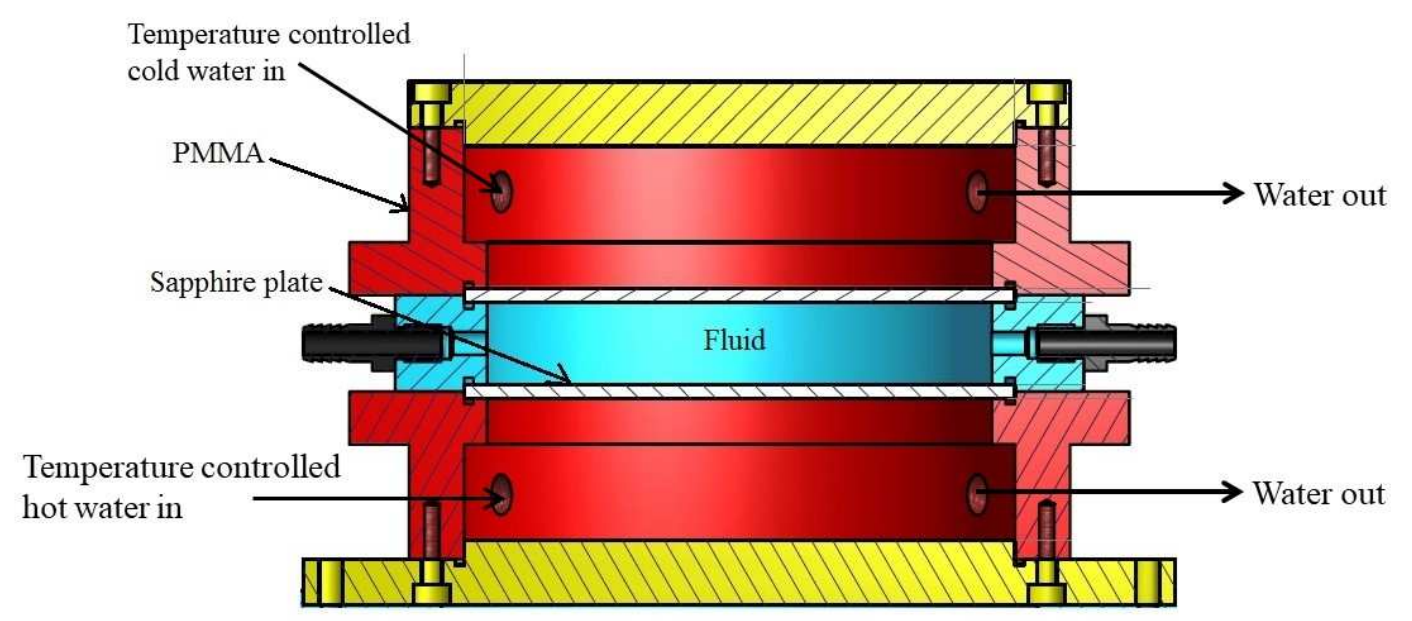

FIG. 7. Cross-section of the experimental apparatus: the fluid layer lies between two sapphire plates.

not perfect and has some drawbacks [37], however, it helps to have good results. For the characteristics of the optical arrangement see table I.

\section{B. Physical properties of the fluid}

Two types of fluids are used. The first one is a glycerol solution. It is used as a Newtonian fluid for the validation of the experimental set-up. The second one corresponds to aqueous xanthan gum solutions at different concentrations; these are shear-thinning fluids.

To study the rheological behavior of each fluid, an AR2000 rheometer is used with a cone and plate geometry characterized by a diameter of $60 \mathrm{~mm}$ and an angle of $1.036^{\circ}$. The rheograms are measured at different temperatures to account for the variation of the viscosity with temperature.

\section{Newtonian fluid}

The Newtonian fluid used in this study is an anhydrous glycerol solution, whose thermophysical properties can be found in [38]. Based on the data given in [38], Stengel et al. [39] proposed the following relations for the thermal capacity $\hat{C} p$, the density $\hat{\rho}$ and the thermal 


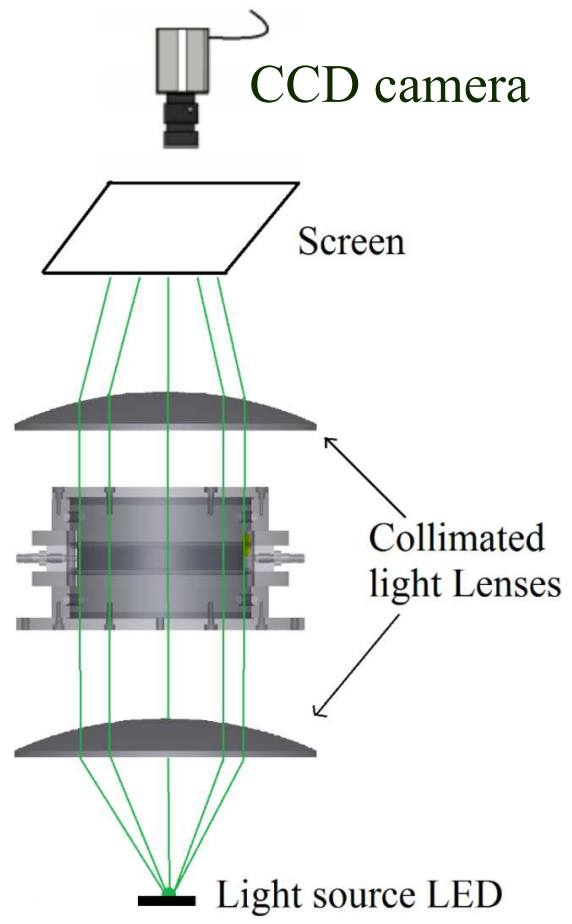

FIG. 8. Optical arrangement for shadowgraph visualizations.

conductivity $\hat{k}$ in the temperature range $\hat{T}=0-50^{\circ} \mathrm{C}$ :

$$
\begin{aligned}
& \hat{C}_{p}=41.866 \times(54+0.15 \times T) \quad\left[\mathrm{J} /\left(\mathrm{kg} .{ }^{\circ} \mathrm{C}\right)\right], \\
& \hat{\rho}=1257\left[1-\hat{\alpha}(\hat{T}-24)-\hat{\beta}(\hat{T}-24)^{2}\right] \quad\left[\mathrm{kg} / \mathrm{m}^{3}\right] \\
& \hat{\alpha}=47.8 \times 10^{-5}\left[1 /{ }^{\circ} \mathrm{C}\right] \text { and } \hat{\beta}=0.2 \times 10^{-5}\left[1 /{ }^{\circ} \mathrm{C}\right]^{2} \\
& \hat{k}=0.286 \quad\left[\mathrm{~W} /\left(\mathrm{m} .{ }^{\circ} \mathrm{C}\right)\right] .
\end{aligned}
$$

The viscosity of glyerol was measured at different temperatures between $10^{\circ} \mathrm{C}$ and $40^{\circ} \mathrm{C}$. In this temperature range, $\hat{\mu}(\hat{T})$ can be fitted by an exponential law:

$$
\hat{\mu}(\hat{T})=\hat{\mu}\left(\hat{T}_{0}\right) \times \exp \left(-\hat{b} \times\left(\hat{T}-\hat{T}_{0}\right)\right),
$$

with

$$
\hat{b}=0.083^{\circ} C^{-1} \text { and } \hat{\mu}\left(\hat{T}_{0}\right)=0.956 \text { Pa.s }
$$

Note that for each rheological measurement, it is important to change the fluid sample in order to prevent that glycerol absorbs moisture from the atmosphere, since it is hygroscopic. The Prandtl number evaluated at the mean temperature $\hat{T}_{0}$ is very large: $\operatorname{Pr}=8064$. 


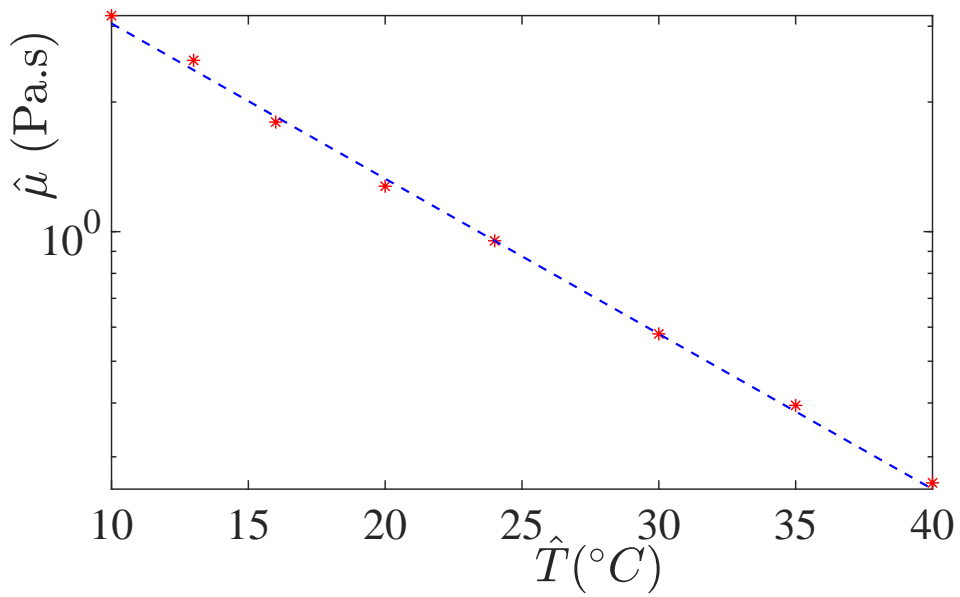

FIG. 9. Variation of the viscosity of the anhydrous glycerol viscosity with temperature $\hat{T}$. The stars are our measurements. The dashed line is the exponential fit Eq.(36)

\section{Shear-thinning fluids}

The shear-thinning fluids used in our experiments are aqueous polymeric solutions of Xanthan gum solutions obeying to the Carreau rheological model. Aqueous solutions were prepared at two concentrations, 1000 ppm and 1200 ppm, by adding Xanthan powder in small amounts, which were measured by an electronic high precision weighing scale, into deionized water. A transparent and homogeneous solution is obtained after few hours of gentle stirring.

The thermo-physical properties of xanthan gum solution 1000 ppm have been measured by means of a calorimeter $(\mu d s c 3-S E T A R A M)$. In the range of temperature $\left[20^{\circ} C, 60^{\circ} \mathrm{C}\right]$, the following relations can be obtained :

$$
\begin{array}{ll}
\hat{C}_{p}=4225.2+0.895 \times T & {\left[\mathrm{~J} /\left(\mathrm{kg} .{ }^{\circ} \mathrm{C}\right)\right],} \\
\hat{k}=0.5153+0.0022 \times T & {\left[\mathrm{~W} /\left(\mathrm{m} .{ }^{\circ} \mathrm{C}\right)\right] .}
\end{array}
$$

As the thermo-physical properties of xanthan solutions vary little in the range of concentrations aforementioned, we have only measured these properties for the concentration of 0.1\%. The density and the thermal expansion coefficient were supposed identical with that of pure water. Based on the data compiled by Mess in (http:/www.thermexcel.com), our 


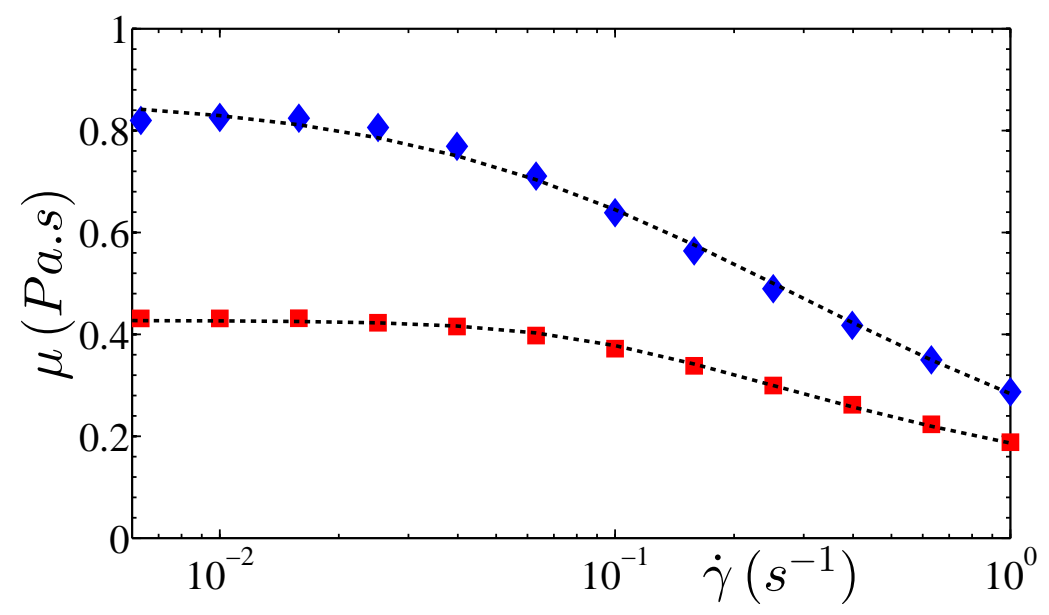

FIG. 10. Flow curves of the xanthan gum solutions at $\hat{T}=24^{\circ} \mathrm{C}$. The dashed lines correspond to the Carreau model given by Eq.42. $\diamond: 1200$ ppm xanthan concentration and $\square$ : 1000 ppm xanthan concentration

best estimate of the density of water in the temperature range $\hat{T}=0-100^{\circ} \mathrm{C}$ is

$$
\begin{aligned}
& \hat{\rho}=997.2965\left[1-\hat{\alpha}\left(\hat{T}-\hat{T}_{0}\right)-\hat{\beta}\left(\hat{T}-\hat{T}_{0}\right)^{2}\right] \quad\left[\mathrm{kg} / \mathrm{m}^{3}\right] \\
& \hat{\alpha}=2.48 \times 10^{-4} \quad\left[1 /{ }^{\circ} \mathrm{C}\right], \quad \hat{\beta}=5.01 \times 10^{-6} \quad\left[1 /{ }^{\circ} \mathrm{C}\right]^{2}
\end{aligned}
$$

Flow curves are displayed in figure 10 for the two xanthan gum concentrations at $\hat{T}=$ $\hat{T}_{0}=24^{\circ} \mathrm{C}$. The shear viscosity $\hat{\mu}$ decreases with increasing the shear rate $\dot{\gamma}$. Xanthan gum solutions can be fitted by the Carreau model

$$
\hat{\mu}=\hat{\mu}_{0}\left(1+(\lambda \dot{\gamma})^{2}\right)^{\frac{n_{c}-1}{2}}
$$

The Carreau rheological parameters are given in Table II. The variation of the viscosity with temperature has been also investigated. It is found at low and moderate shear-rates, the thermodependency of the viscosity can be described by an exponential model:

$$
\hat{\mu}_{0}(\hat{T})=\hat{\mu}_{0}\left(\hat{T}_{0}\right) \exp \left[-\hat{b}\left(\hat{T}-\hat{T}_{0}\right)\right]
$$

The coefficient $\hat{b}$ (Table II) is practically four times smaller than that of the Glycerol. As for the Newtonian fluid considered, the Prandtl number for the shear-thinning fluids used is very large, we have $\operatorname{Pr}=3214$ and 6129 for xanthan gum at $1000 \mathrm{ppm}$ and $1200 \mathrm{ppm}$, respectively. 


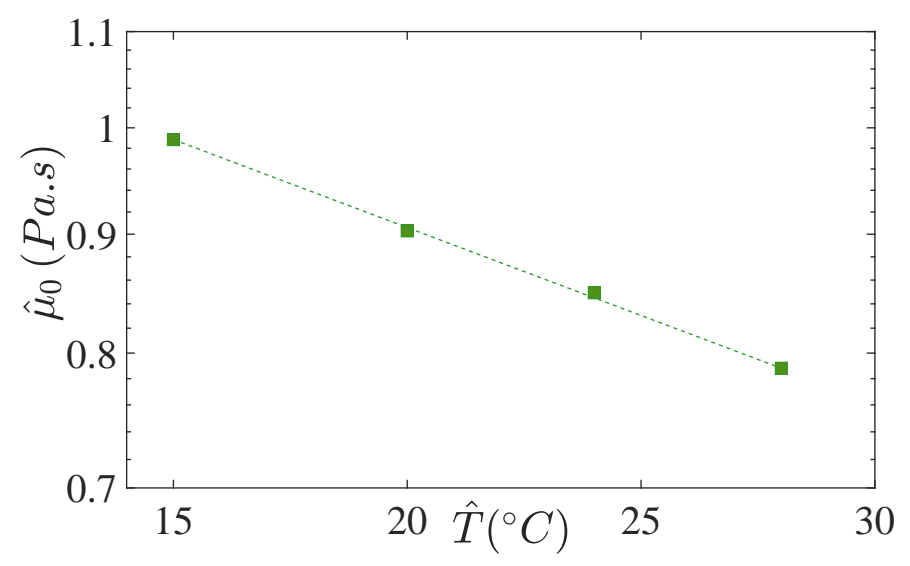

FIG. 11. Xanthan gum at $1200 \mathrm{ppm}$. Variation of $\hat{\mu}_{0}$ as a function of temperature. ( $\square$ ) Experimental data. (-) Exponential fit.

\begin{tabular}{ccccccc}
\hline concentration & $n_{c}$ & $\hat{\lambda}(\mathrm{s})$ & $\hat{\mu}_{0}(\mathrm{~Pa} . \mathrm{s})$ & $\hat{b}\left({ }^{\circ} \mathrm{C}^{-1}\right)$ & $S_{t h}(A R=4)$ & $S_{t h}(L=3)$ \\
$1000 \mathrm{ppm}$ & 0.38 & 9.9 & 0.43 & $2.2 \times 10^{-2}$ & $1.1 \times 10^{-5}$ & $0.35 \times 10^{-5}$ \\
$1200 \mathrm{ppm}$ & 0.36 & 17.2 & 0.82 & $2.25 \times 10^{-2}$ & $3.4 \times 10^{-5}$ & $1.05 \times 10^{-5}$ \\
\hline
\end{tabular}

TABLE II. Carreau rheological parameters for xanthan gum solutions.

\section{EXPERIMENTAL RESULTS}

In our experiments, the temperature difference $\Delta \hat{T}$ between the top and bottom walls is increased by keeping constant the mean temperature $\hat{T}_{0}$.

\section{A. Newtonian fluid}

Experiments were made using an anhydrous glycerol solution. The thickness of the fluid layer is $\hat{d}=15 \mathrm{~mm}$ giving an aspect ratio $L=4$. At the onset of convection, the temperature difference between the top and the bottom walls is $\Delta \hat{T}_{c, \exp }=7.9 \pm 0.1^{\circ} \mathrm{C}$, and the critical Rayleigh number $R a_{c, \text { exp }}=1742 \pm 20$. The viscosity ratio $r$, the coefficients $\gamma_{i}$ and the Busse parameter are: $r_{c}=1.92, \gamma_{0 c}=3.84 \times 10^{-3}, \gamma_{1 c}=0.033, \gamma_{2 c}=-0.67, \gamma_{4 c}=0.02$ and $Q_{c}=-2.18$, respectively. The extent of departure from Boussinesq approximations is caused mainly by the variation of the viscosity with temperature.

For an infinitely extended fluid layer with $r=1.92$ and $\gamma_{1}=0.033$, the linear theory gives 
$R a_{c}(\infty)=1717$. For a cylindrical geometry with insulated lateral walls, Charlson\& Sani [17] have shown that $R a_{c}(L)$ is given by

$$
R a_{c}(L)=\frac{\pi^{2} \zeta_{0}^{2}}{4 L^{2}} R a_{c}(\infty)+R a_{c}(\infty)
$$

with $\zeta_{0}^{2}=0.149$. In our case, we obtain $R a_{c}(L=4)=1756$. An additional correction due to the finite thermal conductivity of the top and bottom plates: the ratio of conductivities of sapphire and water is $\sigma \approx 120$. Using figure 1 given in [23], we obtain a correction $\Delta R a_{c}=-6$. Therefore, taking into account the corrections due to the finite aspect ratio and finite thermal conductivity of the top and bottom walls we obtain $R a_{c, t h}=1750$. The relative difference between $R a_{c, t h}$ and $R a_{c, \exp }$ is less than $1 \%$.

Figure 12 shows shadowgraph pictures obtained for different values of the temperature difference across the fluid layer. Close to the onset at $R a=1809$, irregular convection patterns are observed (figure 12(2)). One may distinguish rolls and squares. In the case of an infinite aspect ratio with the assumption that the viscosity varies linearly with temperature, Busse \& Frick [40] found that near the critical conditions, rolls are preferred for low values of $r$, but squares are preferred for large values of $r$. The change from rolls to squares occurs at $r \approx 2$, which is close our experimental value. It might explain the occurence of irregular patterns. For larger $R a$, figure 12(3)-(4) convection occurs in form of rolls. Note that the rolls has a tendency to form an "arch", they terminate with their axis perpendicular to the side wall. This configuration is well known in the literature [41, 42] and observed in different geometries when the ratio between the thermal conductivities of the sidewalls and that of the fluid is close to 1 [42]. We have also noticed a slight decrease of the wavenumber with increasing $R a$ in agreement with the literature [6], [7] [5] and [8]. For instance, we have obtained $k=3.4 \pm 0.1$ at $R a=2440$ and $k=3.2 \pm 0.1$ at $R a=2670$.

\section{B. Shear-thinning fluid}

1. Xanthan gum solution at $1200 \mathrm{ppm}$ with a fluid layer depth $15 \mathrm{~mm}(L=4)$

For the xanthan gum solution at $1200 \mathrm{ppm}$ with $\hat{d}=15 \mathrm{~mm}$, the shear-thinning degree is $S_{t h}=3.4 \times 10^{-5}$. The onset of convection is observed at $\Delta \hat{T}=23.0 \pm 0.1^{\circ} \mathrm{C}$ leading to $R a_{c, \exp }=1704 \pm 8$. The viscosity ratio, the coefficients $\gamma_{i}$ and the Busse parameter are: $r_{c}=1.68, \gamma_{0 c}=5.86 \times 10^{-3}, \gamma_{1 c}=0.46, \gamma_{2 c}=-0.55, \gamma_{3 c}=0.09, \gamma_{4 c}=5 \times 10^{-3}$ 

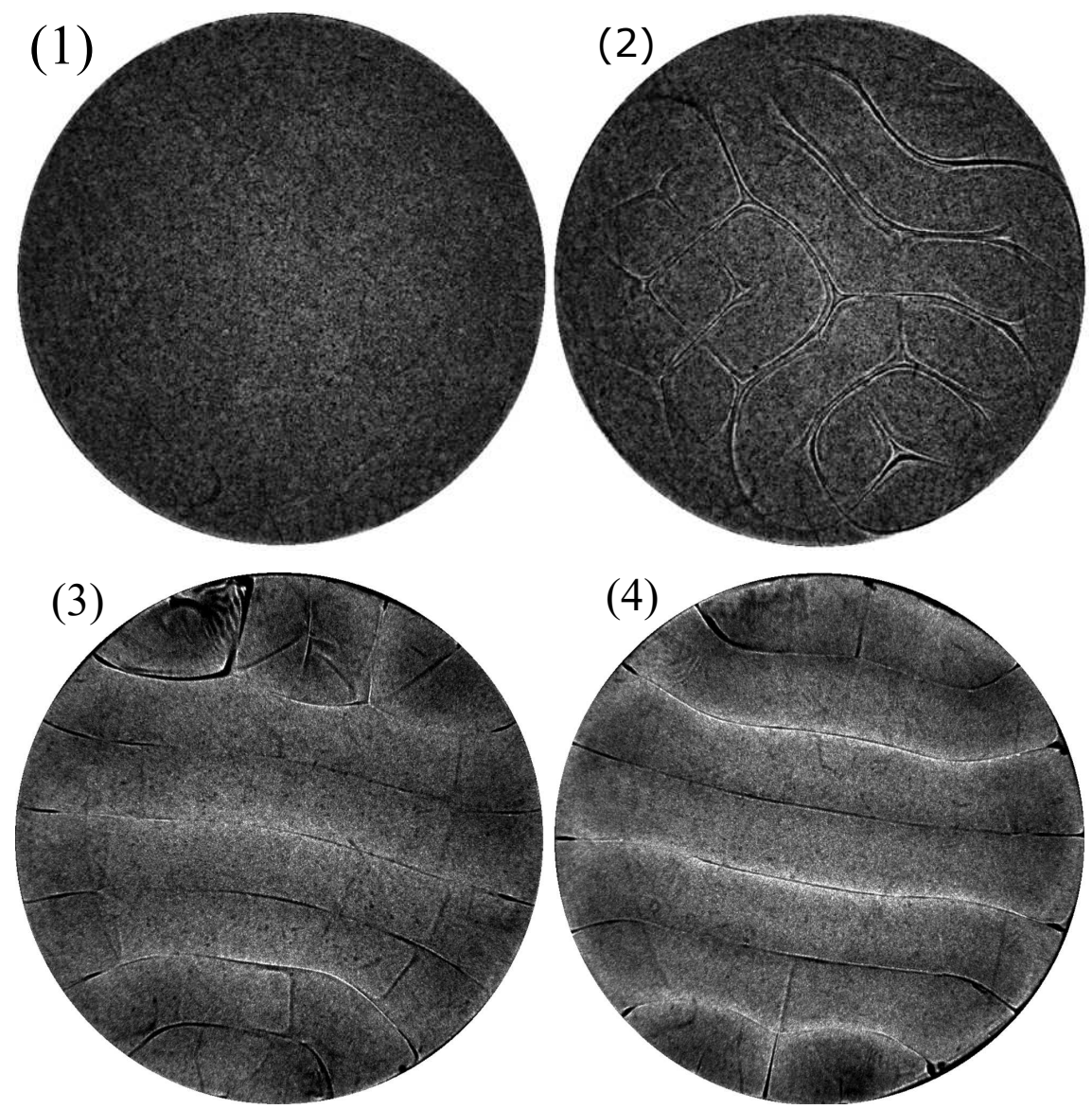

FIG. 12. Glycerol with $\hat{d}=15 \mathrm{~mm}$. Shadowgraph pattern in the $15 \mathrm{~mm}$ deep layer at different values of the temperature difference between the top and bottom walls. (1) $\Delta \hat{T}=7.8^{\circ} \mathrm{C}, R a=1720 ;(2)$ $\Delta \hat{T}=8.2^{\circ} \mathrm{C}, R a=1809, Q=-2.26$; (3) $\Delta \hat{T}=12.0^{\circ} \mathrm{C}, R a=2646, Q=-3.38 ;(4): \Delta \hat{T}=16.3^{\circ} \mathrm{C}$, $R a=3594, Q=-4.73$.

and $Q_{c}=-3.90$ respectively. The departure from the Boussinesq approximation is mainly induced by the variation of the viscosity and thermal expansion with temperature.

From theoretical point of view, the critical Rayleigh number for an infinite extended fluid layer, with $r=1.68$ and $\gamma_{1}=0.49$, is $R a_{c}(\infty)=1694$. Taking into account the corrections due to the finite size of the experimental cell and the finite thermal conductivity of the top and bottom walls, we obtain $R a_{c, t h}=1720$. The relative difference between $R a_{c, t h}$ and $R a_{c, \text { exp }}$ is about $1 \%$.

Figure 13 shows shadowgraph pictures obtained when the temperature difference across the fluid layer increases above threshold. The central dark spot corresponds to ascending hot fluid. The white contours outlining the hexagonal cells correspond to descending cold 
fluid. They are brighter with increasing $\Delta \hat{T}$, i.e. with increasing the intensity of convection. Hexagons are observed for all the range of $\Delta \hat{T}$ considered, $23^{\circ} \mathrm{C}<\Delta \hat{T}<40^{\circ} \mathrm{C}$, wich corresponds to $1704 \leq R a \leq 2960$. This is consistent with the large Q values, i.e. strong deviations from Boussinesq approximations. For a fluid layer of infinite horizontal extent with parameters $r$ and $\gamma_{1}$ taken at the critical conditions, $r=r_{c}=1.68, \gamma_{1}=\gamma_{1 c}=0.46$ and assumed constant, the weakly nonlinear analysis predicts that hexagons with $k=k_{c}$ ( $q=0$ ) are stable up to $R a_{h, t h}=3642$, whereas the solution in the form of rolls is stable from $R a \geq R a_{r, t h}=2264$.

It is worthy to observe the increase of the number of the cells when the temperature difference increases. An increase of the number of cells is equivalent to a decrease of the cell size or an incease of the wavenumber. The main mechanism whereby the size of the hexagonal cells decreases as $\Delta \hat{T}$ is increased is the nucleation of new cells at the periphery of the fluid layer, where probably the largest non conformity of the vertical temperature gradient occurs. Another mechanism observed is the cellular division (mitosis).

In figure 14, we have reported in the plane $\left(q=k-k_{c}, \epsilon=\left(R a-R a_{c}\right) / R a_{c}\right)$ the measured wavenumber for different values of the distance to the threshold of convection. The experimental data are inside the domain of stable wavenumbers calculated using the parameters $r=1.68, \gamma_{1}=0.46$ and $S_{t h}=3.3 \times 10^{-5}$. It seems that $q=\left(k-k_{c}\right)$ varies linearly with $\epsilon$ between $\epsilon=0$ and $\epsilon \approx 0.8$. The average slope which is indicated by a dashed line on figure 14 is $q=0.47 \epsilon$. It is worthy to note that a wide range of theoretical stable wavenumbers are not selected in practice.

\section{Xanthan gum solution at 1200 ppm with a fluid layer depth $\hat{d}=20 \mathrm{~mm}, L=3$}

With increasing the thickness of the fluid layer, the temperature difference needed for the onset of convection decreases and consequently the departure from Boussinesq approximation is reduced. Hence, for the xanthan gum solution at $1200 \mathrm{ppm}$ with $\hat{d}=20 \mathrm{~mm}$, the onset of convection was observed at $\Delta \hat{T}=11.0 \pm 0.1^{\circ} \mathrm{C}$ which corresponds to $R a_{c, \text { exp }}=1930 \pm 20$. The shear-thinning degree of the fluid is $S_{t h}=1.05 \times 10^{-5}$. The viscosity ratio, the coefficients $\gamma_{i}$ and the Busse parameter are: $r_{c}=1.28, \gamma_{0 c} \approx 3.0 \times 10^{-3}, \gamma_{1 c}=0.25, \gamma_{2 c}=-0.27$, $\gamma_{3 c}=0.046, \gamma_{4 c}=2.5 \times 10^{-3}$ and $Q_{c}=-1.95$ respectively.

For an infinite aspect ratio, with $r=r_{c}=1.28$ and $\gamma_{1}=\gamma_{1 c}=0.25$, the critical Rayleigh 

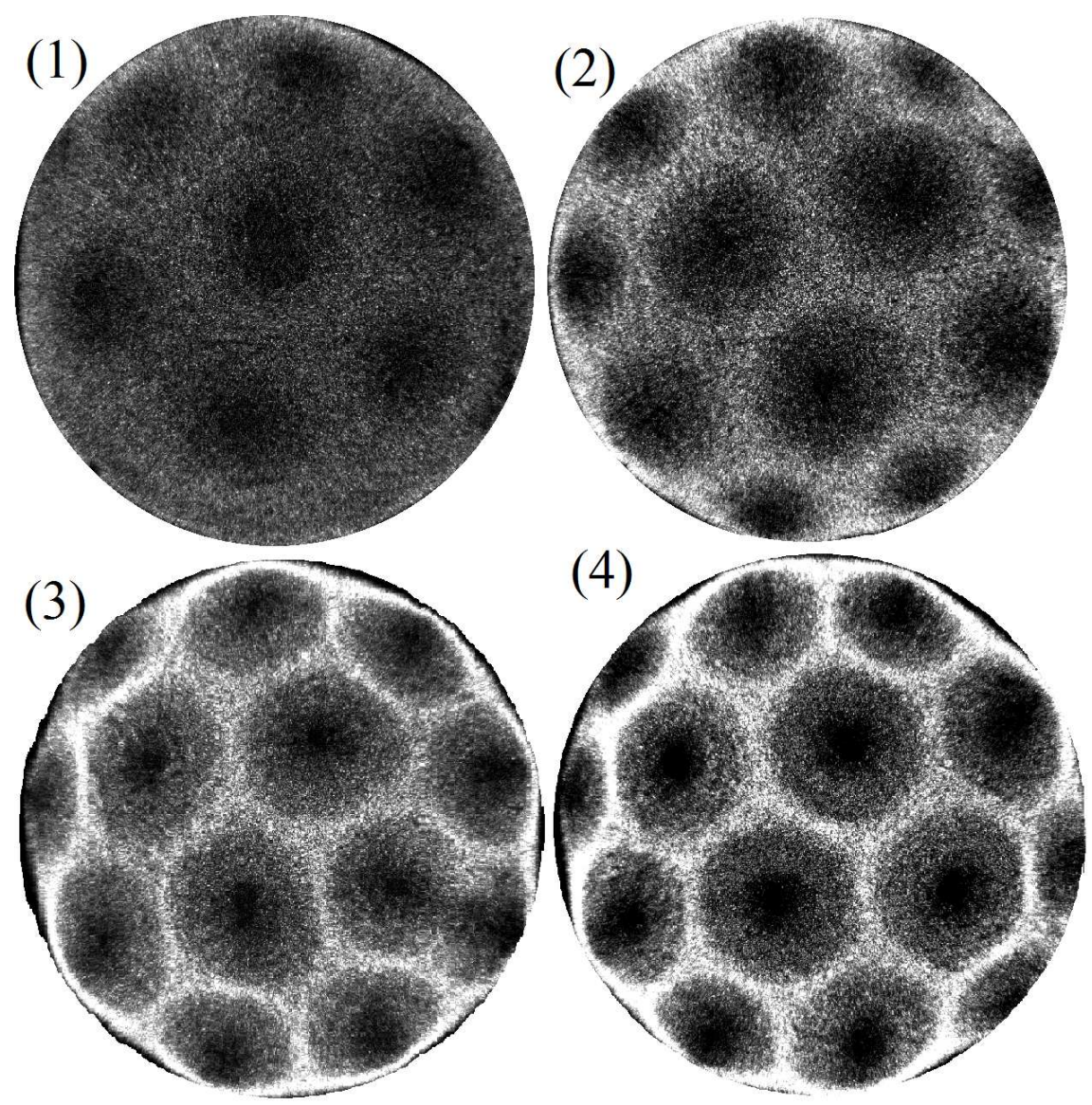

FIG. 13. Xanthan gum solution at $1200 \mathrm{ppm}$. Shadowgraph pattern in the $15 \mathrm{~mm}$ deep layer at different values of the temperature difference between the top and bottom walls. (1) $\Delta \hat{T}=26.0^{\circ} \mathrm{C}$, $R a=1926, Q=-4.21 ;(2) \Delta \hat{T}=31.5^{\circ} \mathrm{C}, R a=2336, Q=-5.26 ;(3) \Delta \hat{T}=38.25^{\circ} \mathrm{C}, R a=2837$, $Q=-6.37 ;(4) \Delta \hat{T}=39.0^{\circ} \mathrm{C}, R a=2890, Q=-6.75$.

number is $R a_{c}(\infty)=1706$. Taking into account the corrections due to the finite size of the experimental cell and the finite thermal conductivity of the top and bottom walls, we obtain $R a_{c, t h}=1769$. The relative difference between $R a_{c, t h}$ and $R a_{c, e x p}$ is about $9 \%$.

Shadowgraph pictures obtained when the temperature difference across the fluid layer increases are shown in figure 15. In the range $R a_{c, \text { exp }}=1930 \leq R a \leq 5047$ convection takes place only in form of hexagons. An illustration is given in figure 15(1). Then further in the range $5047 \leq R a \leq 5400$, hexagons and rolls coexist as it is shown in figures 15(2)-(4). At $R a>5400$ only hexagons are observed. An illustration is given in figure 15(6). Such a sequence: hexagons $\rightarrow$ rolls + hexagons $\rightarrow$ hexagons was never observed in the case of liquids. This restabilization of hexagons which can be termed as "reentrant hexagons", [19] 


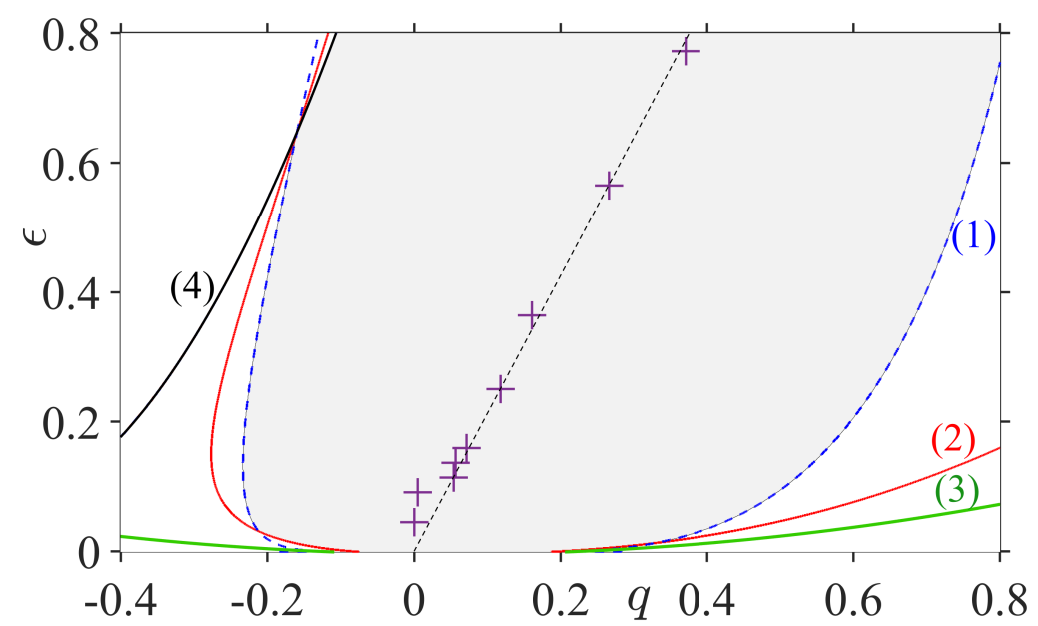

FIG. 14. Xanthan gum solution at $1200 \mathrm{ppm}$ with $\hat{d}=15 \mathrm{~mm}$. Hexagon stability diagram for $r=1.68, \gamma_{1}=0.46$ and $S_{t h}=3.3 \times 10^{-5}$. Curve (1) $D_{\perp}=0$, curve (2) $D_{\|}=0$, curve (3) bifurcation from the conductive state to convection with hexagons, curve (4) bifurcation from hexagons to rolls. $(+)$ Experimental data: variation of $q=\left(k-k_{c}\right)$ in the range of $\epsilon$ values considered.

is a consequence of the increase of the strength of non-Boussinesq effects with increasing the temperature difference, i.e. with increasing Rayleigh number [18]. In figure 16(a) we have represented in the plane $(q, \epsilon)$ the domain of stable wavenumbers predicted by the weakly nonlinear theory using the parameters $r$ and $\gamma_{1}$ evaluated at the critical conditions. We have reported our experimental results. Stable hexagons are observed outside the domain of stability predicted by the theory. This discrepancy is a consequence on one hand of the finite aspect ratio of the experimental setup and on the other hand, in the weakly nonlinear analysis, the stability domain of hexagons is determined using the viscosity ratio and the coefficient $\gamma_{1}$ evaluated at the critical conditions, i.e. the increase of these parameters with increasing $\Delta \hat{T}$ is not taken into account.

In figure 16(b), we have reported our experimental data for all the range of the values of $\epsilon$ considered. It seems that for $\epsilon$ between $\epsilon=0$ and $\epsilon \approx 1.5$, the difference $q=k-k_{c}$ increases linearly with $\epsilon$ as $q=0.15 \epsilon$. This increase is weaker than that found in the previous case, because the strength of non-Boussinesq effects is smaller. However, for $1.8<\epsilon<2.4$ $(5400<R a<6700)$, the size of hexagonal cells remains practically constant. 

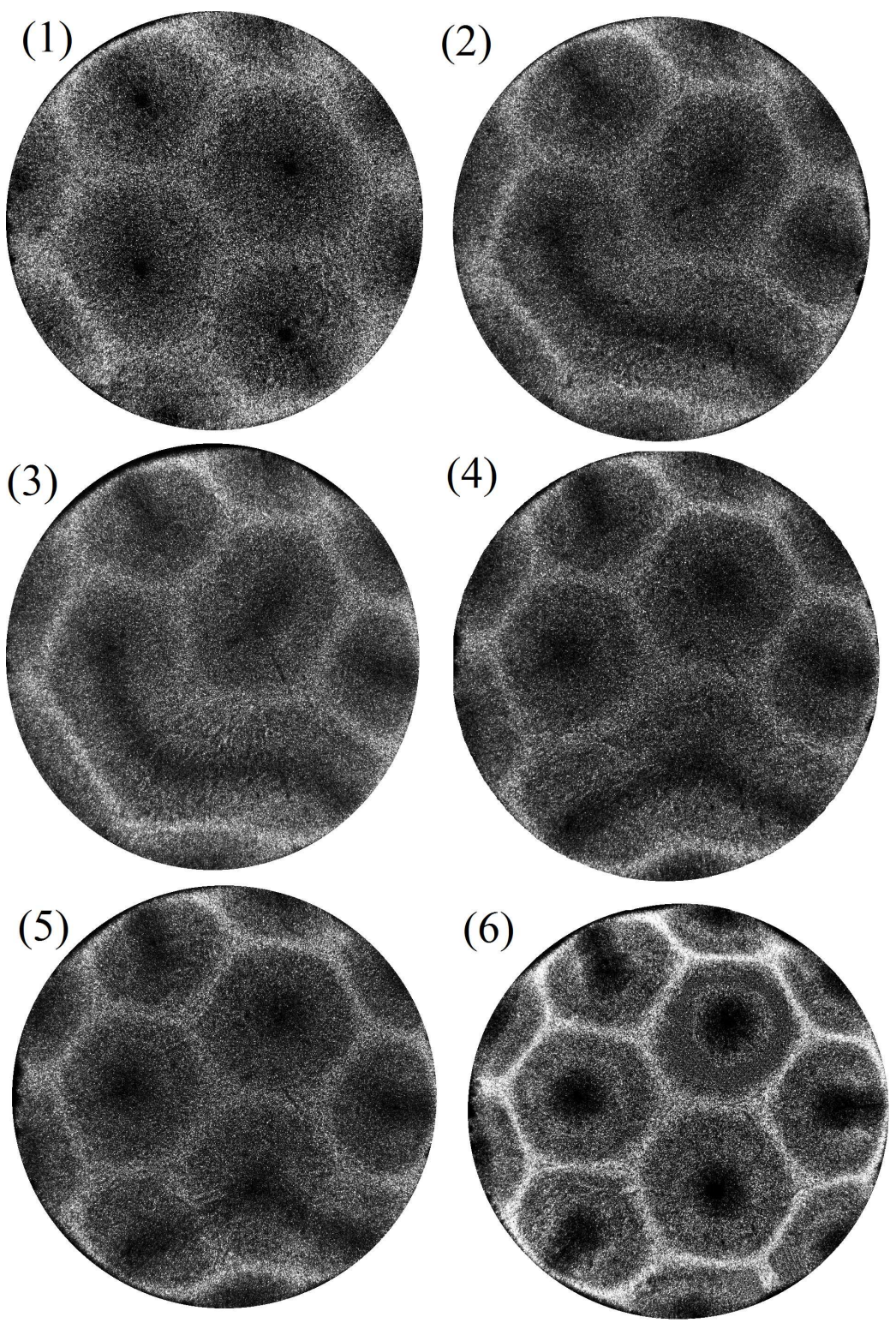

FIG. 15. Xanthan gum solution at $1200 \mathrm{ppm}$. Shadowgraph patterns in the $20 \mathrm{~mm}$ deep layer $(L=3)$ at different values of the temperature difference between the top and bottom walls. (1) $\Delta \hat{T}=24.5^{\circ} \mathrm{C}, R a=4302, Q=-3.90 ;(2) \Delta \hat{T}=29^{\circ} \mathrm{C}, R a=5092, Q=-4.95 ;(3) \Delta \hat{T}=29.5^{\circ} \mathrm{C}$, $R a=5181, Q=-4.95 ;(4) \Delta \hat{T}=30.5^{\circ} \mathrm{C}, R a=5356, Q=-4.95 ;(5) \Delta \hat{T}=31^{\circ} \mathrm{C}, R a=5443.7$, $Q=-5.31 ;(6) \Delta \hat{T}=38^{\circ} \mathrm{C}, R a=6673, Q=-6.42$.

3. Xanthan gum solution at 1000 ppm with a fluid layer depth $\hat{d}=15 \mathrm{~mm}, L=4$

In the case of xanthan gum solution at $1000 \mathrm{ppm}$ with $\hat{d}=15 \mathrm{~mm}$, the onset of convection was observed at $\Delta \hat{T}_{c, \exp }=(12.4 \pm 0.1)^{\circ} \mathrm{C}$, leading to $R a_{c, \exp }=1752 \pm 14$. The 


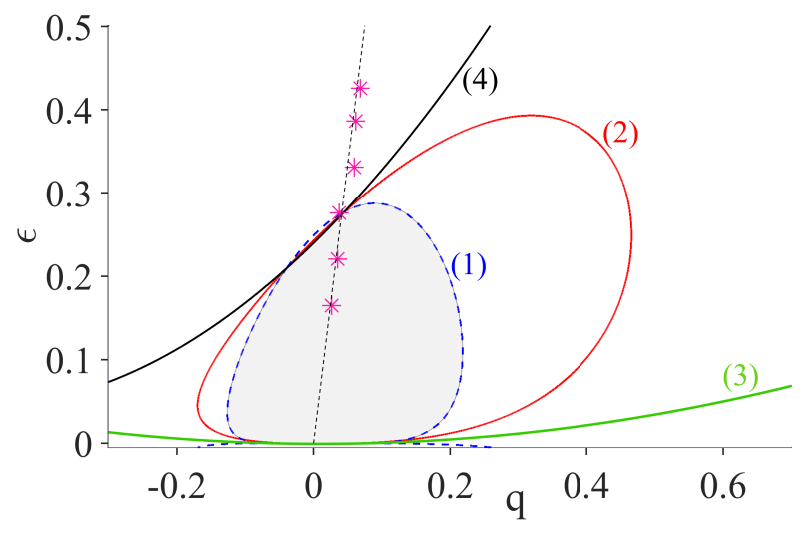

(a)

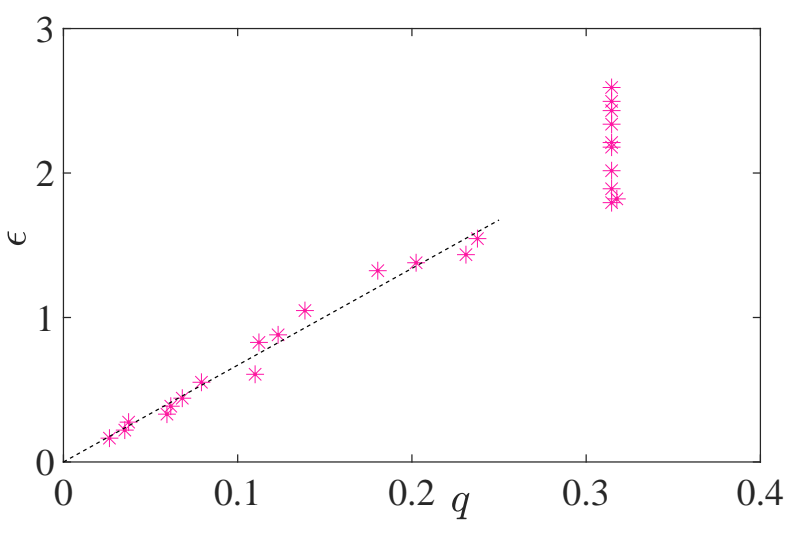

(b)

FIG. 16. Xanthan gum solution at $1200 \mathrm{ppm}$ with $\hat{d}=20 \mathrm{~mm}$. (a) Hexagon stability diagram for $r=1.28, \gamma_{1}=0.25$ and $S_{t h}=1.05 \times 10^{-5}$. Curve (1) $D_{\perp}=0$, curve $(2) D_{\|}=0$, curve (3) bifurcation from the conductive state to convection with hexagons, curve (4) bifurcation from hexagons to rolls. $(+)$ Experimental data: $\left(q=\left(k-k_{c}\right)\right)$ as a function of the distance to the threshold of convection. (b) Experimental variation of $q$ in all the range of $\epsilon$ considered.

viscosity ratio, the coefficients $\gamma_{i}$ and the Busse parameter are: $r_{c}=1.31, \gamma_{0 c} \approx 3.0 \times 10^{-3}$, $\gamma_{1 c}=0.25, \gamma_{2 c}=-0.265, \gamma_{3 c}=0.046, \gamma_{4 c}=2.5 \times 10^{-3}$ and $Q_{c} \approx-1.94$, respectively. The shear-thinning degree evaluated at the onset of convection is $S_{t h}=1.1 \times 10^{-5}$.

For an infinite aspect ratio, with $r=1.31$ and $\gamma_{1}=0.25$, the linear theory gives $R a_{c}(\infty)=1703$.

Taking into account the corrections due to the finite aspect ratio and finite thermal conductivity of the top and bottom wall, we obtain $R a_{c, t h}=1729$. The relative difference between $R a_{c, t h}$ and $R a_{c, \exp }$ is about $1 \%$.

Figure 17 shows shadowgraph pictures obtained when the temperature difference across the fluid layer increases above threshold. In figure 17(1) $(R a=2331)$, hexagonal patterns are observed. From $R a=R a_{r, \exp } \approx 2910$ and with increasing $R a$, rolls invade progressively the cell as it is shown in figures 17(2)-(3). It is observed that the rolls develop from the lateral wall. In a quite small range of Rayleigh numbers, $R a_{r, \exp }=2910<R a<R a_{h, \exp } \approx 3376$, stable coexistence of hexagons and rolls is observed. From $R a=R a_{h \text {,exp }}$, only rolls are observed as it is illustrated in figure 17(4). As indicated previously, the rolls end with their axis perpendiculat to to the lateral wall. The values of $R a_{r}, R a_{h}$ as well as the ratio $R a_{h} / R a_{r}$ are reported in Table III. The theoretical values of the thresholds $R a_{r}$ and $R a_{h}$ are smaller 


\begin{tabular}{ccccc}
\hline Method & $R a_{c}$ & $R a_{r}$ & $R a_{h}$ & $\left(R a_{h}-R a_{r}\right) / R a_{c}$ \\
Theory & 1729 & 1905 & 2338 & 0.25 \\
Experiment & 1752 & 2910 & 3376 & 0.26 \\
\hline
\end{tabular}

TABLE III. Xanthan gum solution at $1000 \mathrm{pppm}$ with $\hat{d}=15 \mathrm{~mm}$. Theoretical and experimental values of $R a_{c}, R a_{r}, R a_{h}$ and $\left(R a_{h}-R a_{r}\right) / R a_{c}$.

than the experimental ones. This is probably a consequence of the finite aspect ratio of the experimental cell. Surprisingly, the theoretical and experimental values of $\left(R a_{h}{ }^{u} R a_{r}\right) / R a_{c}$ which represent the domain where rolls and hexagons compete are quite close.

The stability diagram of hexagons for the corresponding parameters is shown in figure 18 and we have reported our experimental data. Stable hexagons are observed outside the theoretical domain of stability. As in the previous case, this discrepancy could be related to the finite aspect ratio of the experimental setup and to the fact that the increase of non-Boussinesq effects as the temperature difference increases above the onset is not taken in the theoretical study. For $\epsilon$ between $\epsilon=0$ and $\epsilon=0.6$, the difference $q=k-k_{c}$ varies as: $q=0.07 \epsilon$.

To summarize, we have represented in figure 19 the variation of the wavenumber $\left(k-k_{c}\right)$ as a function of the distance to the onset of convection $\epsilon$ for the three cases considered. The wavenumber increases with increasing $\epsilon$ and this increase is all the more significant as nonBoussinesq effects are stronger. Comparison of curve (2) and (3) indicate that for similar non-Boussinesq effects at the onset of convection, the variation of the wavenumber is more significant when the shear-thinning behavior of the fluid is more pronounced, i.e. smaller $n_{c}$ and smaller the characteristic shear-rate $(1 / \lambda)$ for the transition from the Newtonian plateau to the shear-thinning regime.

\section{CONCLUSION}

Rayleigh-Bénard convection in shear-thinning fluids with temperature-dependent physical properties is studied.

- The principal result of this study is the experimental evidence of the increase of the 

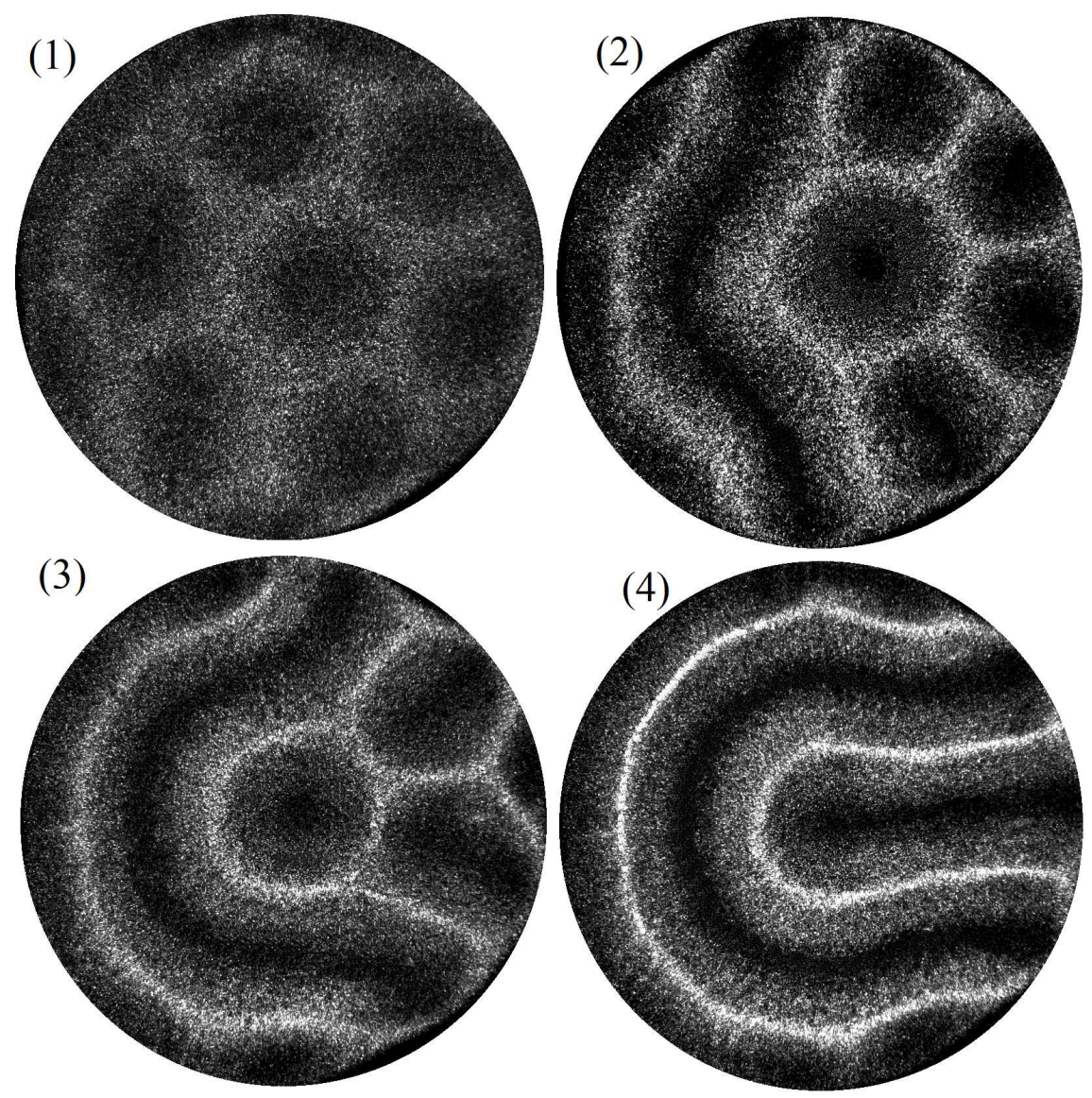

FIG. 17. Xanthan gum solution at $1000 \mathrm{ppm}$ with $\hat{d}=15 \mathrm{~mm}$. Shadowgraph pattern in the $15 \mathrm{~mm}$ deep layer at different values of the temperature difference between the top and bottom walls. (1) $\Delta \hat{T}=16.5^{\circ} \mathrm{C}, R a=2331, Q=-2.55$; (2) $\Delta \hat{T}=21.1^{\circ} \mathrm{C}, R a=2980, Q=-3.53$; (3) $\Delta \hat{T}=22.9^{\circ} \mathrm{C}$, $R a=3235, Q=-3.53 ;(4) \Delta \hat{T}=25.8^{\circ} \mathrm{C}, R a=3645, Q=-4.21$.

wavenumber of the hexagonal cells as the Rayleigh number is increased above the critical value. Furthermore, the increase of the wavenumber with $R a$ is all the more significant as non-Boussinesq effects are stronger. These results provide an experimental support to the theoretical study of Vare et al. [29] that we have extended here to take into account the variation of the thermal expansion coefficient with temperature in addition to that of viscosity. It is demonstrated theoretically that the band of stable wavenumbers is asymmetric and bent towards large wavenumbers.

- The experimental results seem to indicate that $q=\left(k-k_{c}\right)$ increases linearly with $\epsilon$. It appears also that for similar non-Boussinesq effects at the onset of convection, the slope of the variation of $q$ is larger when the shear-thinning behavior is more pronounced, i.e. lower 


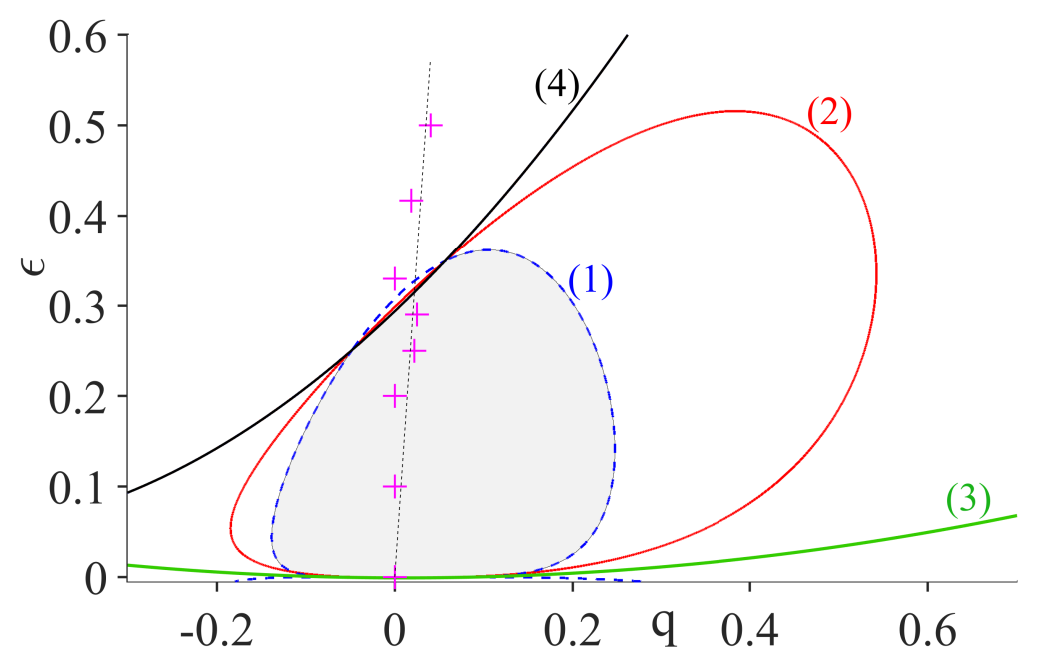

FIG. 18. Xanthan gum solution $1000 \mathrm{ppm}$ with $\hat{d}=15 \mathrm{~mm}$. Hexagon stability diagram for $r=$ 1.31, $\gamma_{1}=0.25$ and $S_{t h}=1.1 \times 10^{-5}$. Curve (1) $D_{\perp}=0$, curve (2) $D_{\|}=0$, curve (3) bifurcation from the conductive state to convection with hexagons, curve (4) bifurcation from hexagons to rolls. $(+)$ Experimental data: $\left(k-k_{c}\right)$ as a function of the distance to the onset of convection.

$n_{c}$ and larger $\lambda$.

- The main mechanism whereby the size of the hexagonal cells decreases as the temperature difference increases is the nucleation of new hexagonal cells at the periphery of the fluid layer. Another mechanism observed experimentally is the cellular division.

- One can note that the increase of the wavenumber of hexagons is in contrast to the wellestablished decrease of the wavenumber of rolls in the supercritical convection [5-7]. This decrease is nevertheless quite weak for fluids with large Prandtl number.

Although, we have been able to make a number of interesting experimental observations, we feel that there is a need for a long series of investigations for various shear-thinning fluids, aspect ratios and Prandtl number. Additional experiments are also needed to investigate the phenomena of "re-entrant hexagons" and the coexistence of rolls and hexagons observed experimentally as well as the uniqueness of the wavenumber. It might also be interesting to analyze how the balance between the kinetic energy dissipated by viscosity and the energy released by buoyancy is altered with increasing the temperature difference. This analysis might provide information to explain why the number of hexagonal cells increases. 


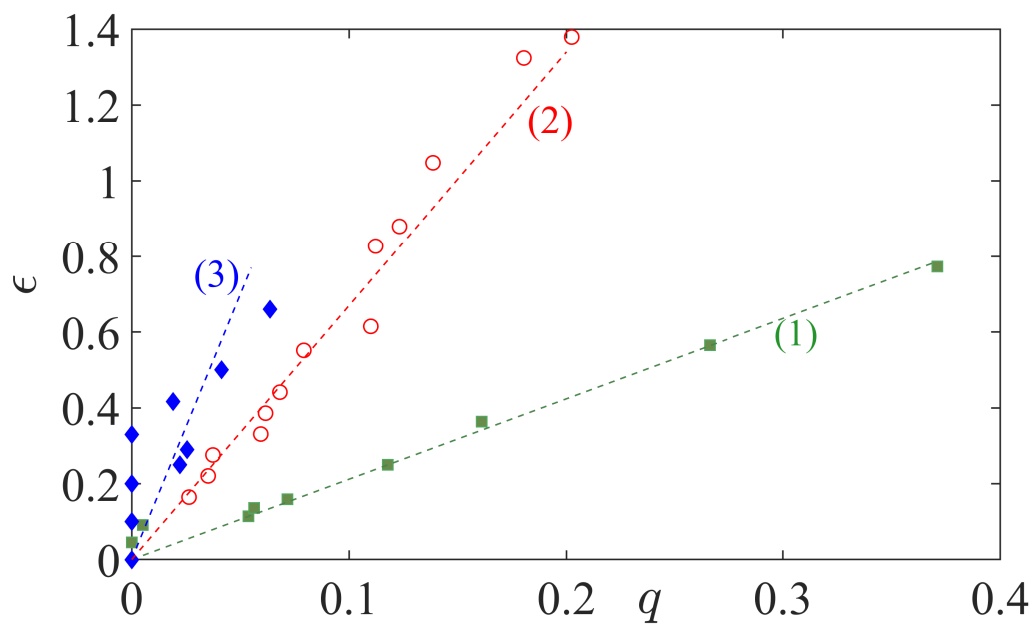

FIG. 19. Variation of $\left(k-k_{c}\right)$ as a function of $\epsilon$. (1) Xanthan gum at $1200 \mathrm{ppm}, \hat{d}=15 \mathrm{~mm}$, $Q_{c}=-3.90 ;(2)$ xanthan gum at $1200 \mathrm{ppm}, \hat{d}=20 \mathrm{~mm}, Q_{c}=-1.95$; (3) xanthan gum at 1000 ppm, $\hat{d}=15 \mathrm{~mm}, Q_{c}=-1.94$.

Finally, from practical point of view, it is interesting to note that Rayleigh-Bénard convection in thin polymer films with thermodependent properties can be harnessed in elegant ways to pattern surfaces [43]. The determination of the wavelength selected by the system is of paramount importance in the control of thin film patterning process. 


\section{Appendix A: Correlations proposed by Busse [9] for a Newtonian fluid}

Assuming a linear variation of the viscosity with temperature, the following correlations for $\epsilon_{a}, \epsilon_{r}$ and $\epsilon_{h}$ are proposed by Busse [9]. The revised version of these correlations given by Bodenschatz et al. [1] is used here:

$$
\epsilon_{a}=-Q^{2} /\left(4 R_{h} R a_{c}\right), \quad \epsilon_{r}=3 Q^{2} R_{r} /\left(F^{2} R a_{c}\right), \quad \epsilon_{h}=\left(9 R_{h}-3 F\right) Q^{2} /\left(F^{2} R a_{c}\right)
$$

with

$$
\begin{aligned}
& R_{h}=0.89360+0.04959 / P r+0.06787 / \operatorname{Pr}^{2} \\
& R_{r}=0.69942-0.00472 / P r+0.00832 / \operatorname{Pr}^{2} \\
& F=0.29127+0.08147 / P r+0.08933 / \operatorname{Pr}^{2}
\end{aligned}
$$

\section{Appendix B: Correlations for the coefficients of equation (22)}

The characteristic time for the instability to grow $\tau_{0}$ and the coherence length $\xi_{0}$ are shown in figure 20 as a function of $r$ and for different values of the parameter $\gamma_{1}$. The Prandtl number is fixed to $\operatorname{Pr}=100$. As it can be observed $\tau_{0}$ varies slightly with $r$ and $\gamma_{1}$, for the ranges considered. The coefficient $\zeta$ arises from non-Oberbeck-Boussinesq effects. It increases with increasing the viscosity ratio, since $\zeta \propto \ln (r)$, and with increasing the parameter $\gamma_{1}$ as it is shown in figure 21. The following correlations are proposed for the coefficients of equation (22):

$$
\begin{aligned}
& \tau_{0}=0.0511-1.53 \times 10^{-3} \times \gamma_{1} \ln (r) \\
& \xi_{0}=0.3855-\left(3.77+34.3 \gamma_{1}\right) \times 10^{-4} r-\left(1.688-4.17 \gamma_{1}\right) \times 10^{-4} r^{2} \\
& \zeta=23.9 \gamma_{1}+9.5 \ln (r) \\
& g_{1 N}=\left(257.6+12.05 \gamma_{1}\right)-\left(1.0+22.6 \gamma_{1}\right) r-\left(0.67-2.63 \gamma_{1}\right) r^{2} \\
& g_{1 N N}=\left(1.15-0.15 \gamma_{1}\right) 10^{6}+\left(0.136-2.3 \gamma_{1}\right) 10^{5} r+\left(1.14-2.56 \gamma_{1}\right) 10^{4} r^{2} \\
& g_{2 N}=\left(367.0+24.0 \gamma_{1}\right)-\left(2.0+49.3 \gamma_{1}\right) r+\left(-1.45+5.65 \gamma_{1}\right) r^{2} \\
& g_{2 N N}=\left(1.31-0.16 \gamma_{1}\right) \times 10^{6}+\left(1.4+24.5 \gamma_{1}\right) \times 10^{4} r+\left(1.18-2.74 \gamma_{1}\right) \times 10^{4} r^{2} \\
& \alpha_{1}=16.17 \gamma_{1}+\left(11.28-2.58 \gamma_{1}\right) \ln (r) \\
& \alpha_{2}=11.17 \gamma_{1}-\left(2.5+1.75 \gamma_{1}\right) \ln (r)
\end{aligned}
$$




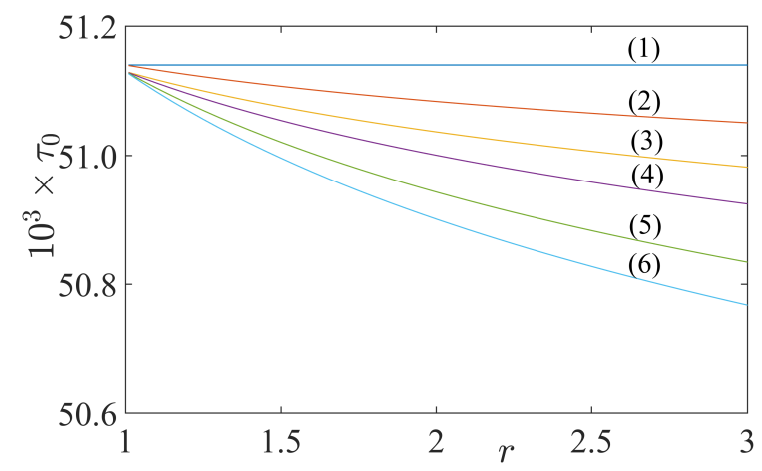

(a)

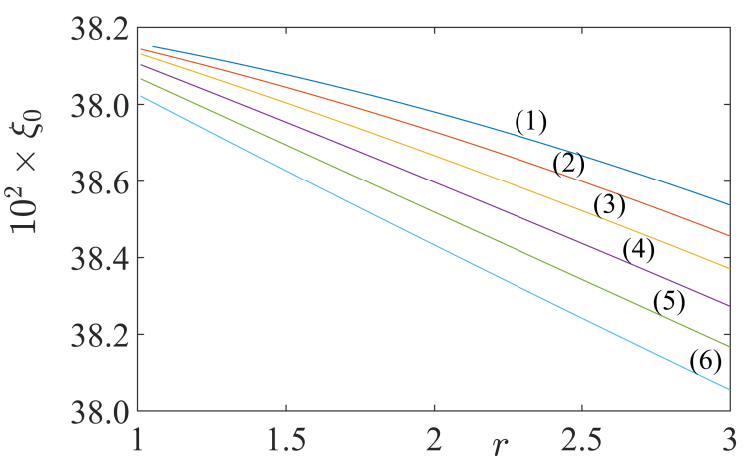

(b)

FIG. 20. Variation of the characteristic time $\tau_{0}$ (a) and the coherence length $\xi_{0}(\mathbf{b})$ as a function of the viscosities ratio, for different values of the parameter $\gamma_{1}$. (1) $\gamma_{1}=0 ;$ (2) $\gamma_{1}=0.1 ;$ (3) $\gamma_{1}=0.2$; (4) $\gamma_{1}=0.3 ;(5) \gamma_{1}=0.4 ;(6) \gamma_{1}=0.5$.

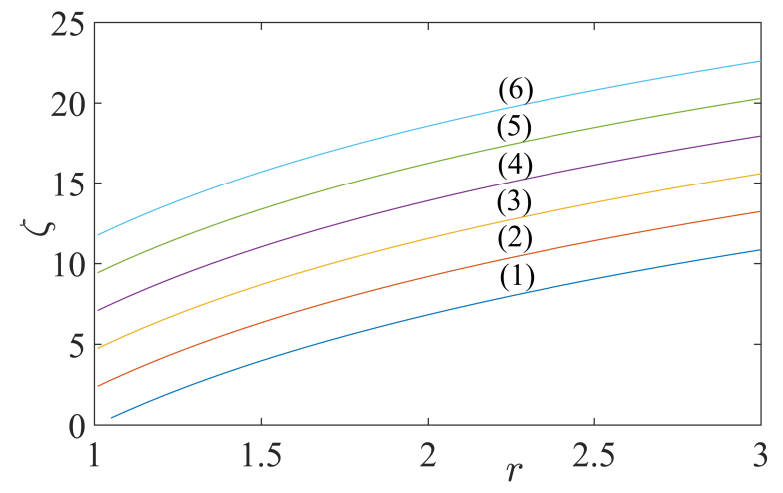

FIG. 21. Variation of $\zeta$ with the viscosity ratio $r$ for different values of the parameter $\gamma_{1}$. The Prandtl number is fixed to $\operatorname{Pr}=100$. (1) $\gamma_{1}=0$; (2) $\gamma_{1}=0.1$; (3) $\gamma_{1}=0.2$; (4) $\gamma_{1}=0.3$; (5) $\gamma_{1}=0.4 ;(6) \gamma_{1}=0.5$

\section{Appendix C: Longitudinal and transverse phase diffusion equations}

The diffusion coefficients $D_{\|}$and $D_{\perp}[29,31,32]$ are given by :

$$
\begin{aligned}
D_{\perp}= & \frac{1}{4} \frac{\xi_{0}^{2}}{\tau_{0}}-\frac{q^{2}}{2 \tilde{u}}\left(\frac{\xi_{0}^{2}}{\tau_{0}}\right)^{2}+\frac{H_{0}^{2}}{8 \tilde{u}}\left(\alpha_{1}-\sqrt{3} \alpha_{2}\right)^{2} \\
D_{\|}= & \frac{3}{4} \frac{\xi_{0}^{2}}{\tau_{0}}-\frac{q^{2}(4 \tilde{u}+\tilde{v})}{2 \tilde{u} \tilde{v}}\left(\frac{\xi_{0}^{2}}{\tau_{0}}\right)^{2}+\frac{H_{0}^{2}}{8 \tilde{u}}\left(\alpha_{1}-\sqrt{3} \alpha_{2}\right)^{2}- \\
& \frac{H_{0}^{2} \alpha_{1}}{\tilde{v}}\left(\alpha_{1}+\sqrt{3} \alpha_{2}\right)+\frac{H_{0} q}{\tilde{v}} \frac{\xi_{0}^{2}}{\tau_{0}}\left(3 \alpha_{1}+\sqrt{3} \alpha_{2}\right),
\end{aligned}
$$




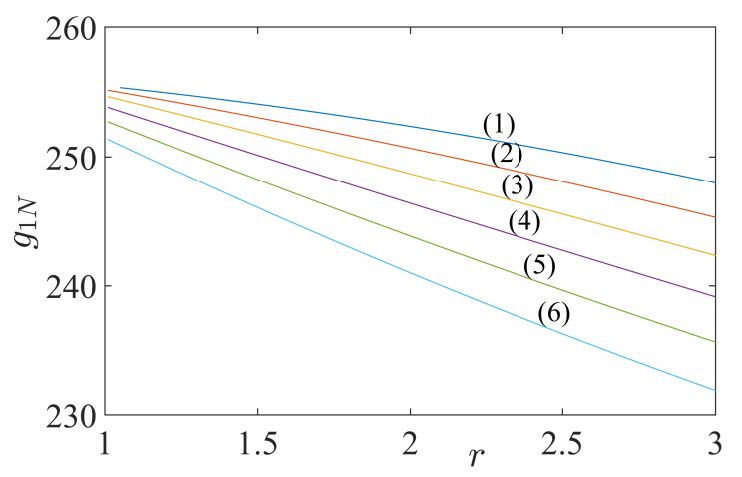

(a)

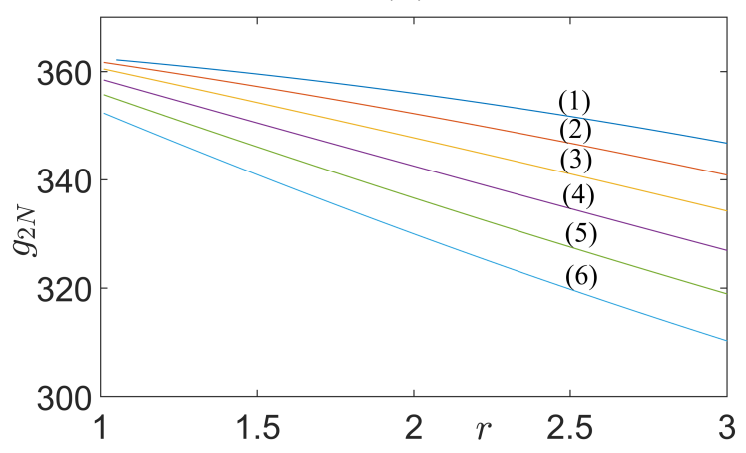

(c)

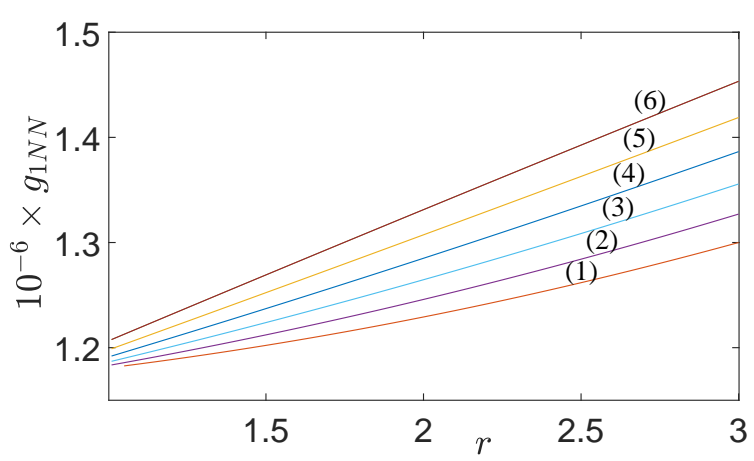

(b)

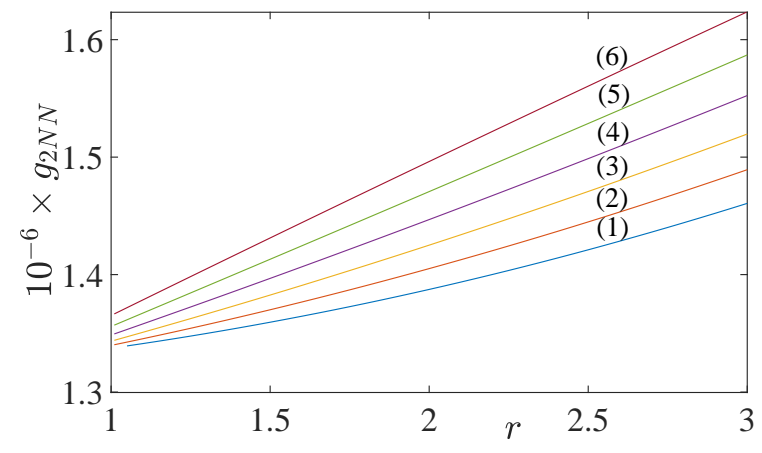

(d)

FIG. 22. (a) "Newtonian" and (b) non-Newtonian contribution to the first Landau coefficient and to the cross-saturation coefficient respectively (c) and (d) as a function of the viscosity ratio $r$ for different values of the parameter $\gamma_{1}$. The Prandtl number is fixed to $\operatorname{Pr}=100$. (1) $\gamma_{1}=0$, (2) $\gamma_{1}=0.1,(3) \gamma_{1}=0.2,(4) \gamma_{1}=0.3,(5) \gamma_{1}=0.4,(6) \gamma_{1}=0.5$.

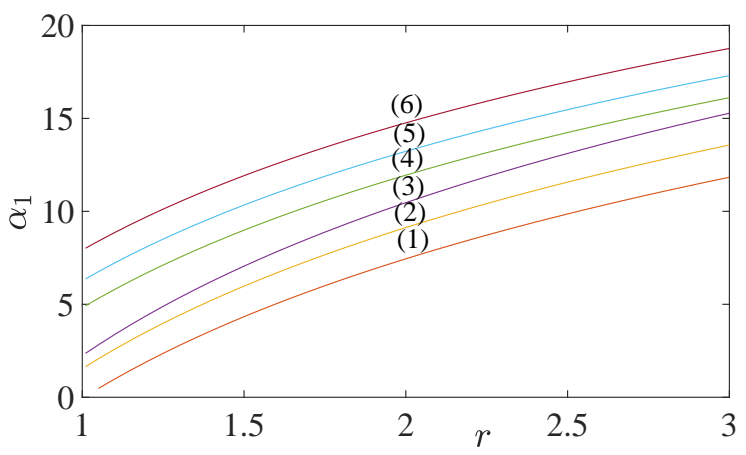

(a)

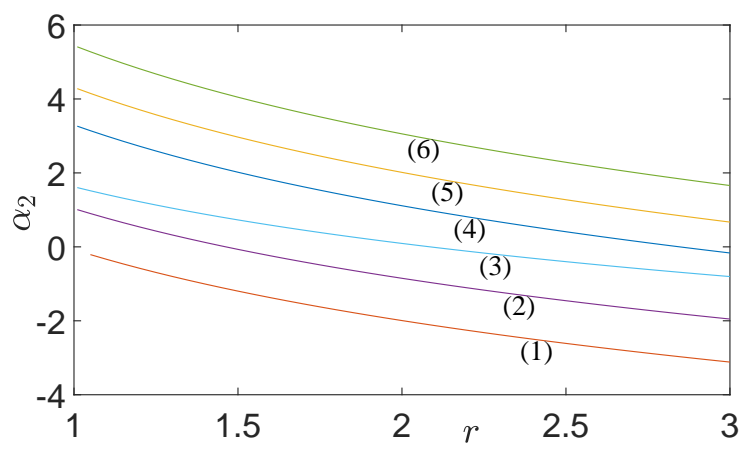

(b)

FIG. 23. Coefficients $\alpha_{1}$ (a) and $\alpha_{2}$ (b) as a function of $r$ for different values of $\gamma_{1}$, with $\operatorname{Pr}=100$.

(1) $\gamma_{1}=0,(2) \gamma_{1}=0.1$, (3) $\gamma_{1}=0.2$, (4) $\gamma_{1}=0.3$, (5) $\gamma_{1}=0.4,(6) \gamma_{1}=0.5$. 
with

$$
\begin{aligned}
& \tilde{u}=H_{0}^{2}\left(g_{1}-g_{2}\right)+\left(\zeta+2 \alpha_{1} q\right) H_{0}, \\
& \tilde{v}=2 H_{0}^{2}\left(g_{1}+2 g_{2}\right)-\left(\zeta+2 \alpha_{1} q\right) H_{0}
\end{aligned}
$$

where, $H_{0}$ is the stationary homogeneous solution of the amplitude equations (22) for hexagons,

$$
H_{0}=\frac{\left(\zeta+2 \alpha_{1} q\right)+\sqrt{\left(\zeta+2 \alpha_{1} q\right)^{2}+4\left(g_{1}+2 g_{2}\right)\left(\epsilon-\xi_{0}^{2} q^{2}\right) / \tau_{0}}}{2\left(g_{1}+2 g_{2}\right)}
$$


[1] E. Bodenschatz, W. Pesch, and G. Ahlers. Recent developments in Rayleigh-Bénard convection. Annu. Rev. Fluid Mech., 32(1):709-778, 2000.

[2] A. Schlüter, D. Lortz, and F. Busse. On the stability of steady finite amplitude convection. J. Fluid Mech., 23(1):129-144, 1965.

[3] F.H. Busse. On the stability of two-dimensional convection in a layer heated from below. J. Math Phys., 46(1-4):140-150, 1967.

[4] F.H. Busse and R. M. Clever. Instabilities of convection rolls in a fluid of moderate Prandtl number. J. Fluid. Mech., 91(2):319-335, 1979.

[5] R. Krishnamurti. On the transition to turbulent convection. part 1. the transition from two-to three-dimensional flow. J. Fluid Mech., 42(2):295-307, 1970.

[6] E.L. Koschmieder. On the wavelength of convective motions. J. Fluid Mech., 35(3):527-530, 1969.

[7] E.L. Koschmieder and S.G. Pallas. Heat transfer through a shallow, horizontal convecting fluid layer. Int. J. Heat Mass Transfer, 17(9):991-1002, 1974.

[8] G.G. Willis, J.W. Deardorff, and R.C.J. Somerville. Roll-diameter dependence in Rayleigh convection and its effect upon the heat flux. J. Fluid Mech., 54(2):351-367, 1972.

[9] F.H. Busse. The stability of finite amplitude cellular convection and its relation to an extremum principle. J. Fluid. Mech, 30(04):625-649, 1967.

[10] E.F.C. Somerscales and T.S. Dougherty. Observed flow patterns at the initiation of convection in a horizontal liquid layer heated from below. J. Fluid Mech., 42(4):755-768, 1970.

[11] C.Q. Hoard, C.R. Robertson, and A. Acrivos. Experiments on the cellular structure in Bénard convection. Int. J. Heat Mass Transfer, 13(5):849-856, 1970.

[12] M. Dubois, P. Berge, and J. Wesfreid. Non-Boussinesq convective structures in water near $4^{\circ}$ C. J. Phys., 39(12):1253-1257, 1978.

[13] G. Ahlers. Effect of departures from the Oberbeck-Boussinesq approximation on the heat transport of horizontal convecting fluid layers. J. Fluid Mech., 98(1):137-148, 1980.

[14] R.W. Walden and G. Ahlers. Non-Boussinesq and penetrative convection in a cylindrical cell. J. Fluid Mech., 109:89-114, 1981.

[15] J.R. de Bruyn E. Bodenschatz, G. Ahlers, and D.S. Cannell. Transitions between patterns in 
thermal convection. Phys. Rev. Lett., 67(22):3078, 1991.

[16] E. Pampaloni, C. Perez-Garcia, L. Albavetti, and S. Ciliberto. Transition from hexagons to rolls in convection in fluids under non-Boussinesq conditions. J. Fluid Mech., 234:393-416, 1992.

[17] G.S. Charlson and R.L. Sani. Thermoconvective instability in a bounded cylindrical fluid layer. Int. J. Heat Mass Transfer, 13(9):1479-1496, 1970.

[18] S. Madruga, H. Riecke, and W. Pesch. Re-entrant hexagons in non-Boussinesq convection. J. Fluid Mech., 548:341-360, 2006.

[19] A. Roy and V. Steinberg. Reentrant hexagons in non-Boussinesq Rayleigh-Bénard convec-tion: Effect of compressibility. Phys. Rev. Lett., 88(24):244503, 2002.

[20] N.J. Balmforth and A.C. Rust. Weakly nonlinear viscoplastic convection. J. Non-Newtonian Fluid Mech., 158:36-45, 2009.

[21] O. Benouared, M. Mamou, and N. Ait-Messaoudene. Numerical nonlinear analysis of subcritical Rayleigh-Bénard convection in a horizontal confined enclosure filled with non-Newtonian fluids. Phys. Fluids, 26(7):073101, 2014.

[22] M. Jenny, E. Plaut, and A. Briard. Numerical study of subcritical Rayleigh-Bénard convection rolls in strongly shear-thinning Carreau fluids. J. Non-Newtonian Fluid Mech., 219:19-34, 2015.

[23] M. Bouteraa and C. Nouar. Weakly nonlinear analysis of Rayleigh-Bénard convection in a non-newtonian fluid between plates of finite conductivity: Influence of shear-thinning effects. Phys. Rev. E, 92(6):063017, 2015.

[24] St. C. Pierre and C. Tien. Experimental investigation of natural convection heat transfer in confined space for non-newtonian fluid. Can. J. Chem. Eng, 41:122-127, 1963.

[25] H-S. Tsuei and C. Tien. Free convection heat transfer in a horizontal layer of non-Newtonian fluid. Can. J. Chem. Eng., 51(2):249-251, 1973.

[26] S.F. Liang and A. Acrivos. Experiments on buoyancy-driven convection in non-Newtonian fluids. Rheol. Acta., 9:447-455, 1970.

[27] M. Darbouli, C. Métivier, S. Leclerc, C. Nouar, M. Bouteera, and D. Stemmelen. Natural convection in shear-thinning fluids: Experimental investigations by MRI. Int. J. Heat Mass Transfer, 95:742-754, 2016.

[28] M. Bouteraa, C. Nouar, E. Plaut, C. Métivier, and A. Kalck. Weakly nonlinear analysis of Rayleigh-Bénard convection in shear-thinning fluids: nature of the bifurcation and pattern 
selection. J. Fluid. Mech, 767:696-734, 2015.

[29] T. Varé, C. Nouar, C. Métivier, and M. Bouteraa. Stability of hexagonal pattern in RayleighBénard convection for thermodependent shear-thinning fluids. J. Fluid Mech., 905, 2020.

[30] R. B. Bird, R. Amstrong, and O. Hassager. Dynamics of polymeric liquids. Wiley-Interscience, New York, 1987.

[31] B. Echebarría and C. Pérez-García. Phase instabilities in hexagonal patterns. Europhys. Lett., 43(1):35, 1998.

[32] B. Echebarría and C. Perez-Garcia. Stability of hexagonal patterns in Bénard-Marangoni convection. Phys. Rev. E, 63(6):066307, 2001.

[33] R. Hoyle. Pattern formation: an introduction to methods. Cambridge University Press, 2006.

[34] J. Lauzeral, D. Metens, and D. Walgraef. On the phase dynamics of hexagonal patterns. Europhys. Lett., 24(9):707, 1993.

[35] B. Pena and C. Perez-Garcia. Stability of Turing patterns in the Brusselator model. Phys. Rev. E, 64(5):056213, 2001.

[36] V.S. Solomatov and C. Jain. Stability range of localized subcritical Rayleigh-Bénard convection in temperature-dependent viscosity fluids: Constraints from two-dimensional simulations. Phys. Fluids, 33(5):056603, 2021.

[37] S.W. Morris S.P. Trainoff J.R. de Bruyn, E. Bodenschatz, Y. Hu, D.S. Cannell, and G. Ahlers. Apparatus for the study of Rayleigh-Bénard convection in gases under pressure. Rev. Sci. Instrum., 67(6):2043-2067, 1996.

[38] A.A. Newman and L.V. Cocks. Glycerol. cleveland. 1968.

[39] K.C. Stengel, D.S. Oliver, and J. R.Booker. Onset of convection in a variable-viscosity fluid. J. Fluid. Mech, 120:411-431, 1982.

[40] F. H. Busse and H. Frick. Square-pattern convection in fluids with strongly temperaturedependent viscosity. J. Fluid. Mech, 150:451-465, 1985.

[41] A.C. Perkins. Mechanisms of instability in Rayleigh-Béenard convection. PhD thesis, Georgia Institute of Technology, 2011.

[42] V. Croquette. Convective pattern dynamics at low Prandtl number: Part II. Contemporary Physics, 30(3):153-171, 1989.

[43] D.W. Janes, J.M. Katzenstein, K. Shanmuganathan, and C.J. Ellison. Directing convection to pattern thin polymer films. J. Polym. Sci. B: Polym. Phys., 51(7):535-545, 2013. 